\title{
Tributylphosphate in the In-Tank Precipitation Process Facilities
}

by

M. J. Barnes

Westinghouse Savannah River Company

Savannah River Site

Aiken, South Carolina 29808

D. T. Hobbs

R. F. Swingle

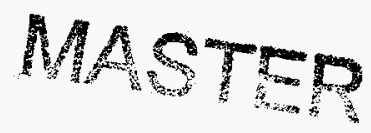

DOE Contract No. DE-AC09-89SR18035

This paper was prepared in connection with work done under the above contract number with the U.S. Department of Energy. By acceptance of this paper, the publisher and/or recipient acknowledges the U.S. Government's right to retain a nonexclusive, royalty-free license in and to any copyright covering this paper, along with the right to reproduce and to authorize others to reproduce all or part of the copyrighted paper. 


\section{DISCLAIMER}

This report was prepared as an account of work sponsored by an agency of the United States Government. Neither the United States Government nor any agency thereof, nor any of their employees, makes any warranty, express or implied, or assumes any legal liability or responsibility for the accuracy, completeness, or usefulness of any information, apparatus, product, or process disclosed, or represents that its use would not infringe privately owned rights. Reference herein to any specific commercial product, process, or service by trade name, trademark, manufacturer, or otherwise does not necessarily constitute or imply its endorsement, recommendation, or favoring by the United States Government or any agency thereof. The views and opinions of authors expressed herein do not necessarily state or reflect those of the United States Government or any agency thereof.

This report has been reproduced directly from the best available copy.

Available to DOE and DOE contractors from the Office of Scientific and Technical Information, P. O. Box 62, Oak Ridge, TN 37831; prices available from (423) 576-8401.

Available to the public from the National Technical Information Service, U. S. Department of Commerce, 5285 Port Royal Road, Springfield, VA 22161. 


\section{DISCLAIMER}

Portions of this document may be illegible in electronic image products. Images are produced from the best available original document. 
WESTINGHOUSE SAVANNAH RIVER COMPANY

SAVANNAH RIVER TECHNOLOGY CENTER
WSRC-AP-93-1162

Keywords:

Decontaminated Salt Solution, Foaming, In-Tank Precipitation, Stripper Columns, Tributylphosphate

Retention Time: Permanent

CC: Distribution

WT-LWG Files, 773-A

TIM, 703-43A

November 23, 1993

To: $\quad$ S. D. Fink, $773-A$

From: M. J. Barnes, 773-A (x5-2104)

D. T. Hobbs, 773-A (x5-2838)

R. F. Swingle, $773-A(\times 5-2369)$

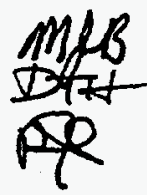

TRIBUTYLPHOSPHATE IN THE IN-TANK PRECIPITATION PROCESS FACILITIES

(U)

\section{SUMMARY}

A material balance investigation and evaluation of $n$-tributylphosphate (TBP) recycle throughout ITP and its carryover to DWPF was performed. Criticality and DWPF-related issues were determined to pose no adverse consequences due to TBP addition. Effects of decomposition products were also considered. Flammability of 1-butanol, a TBP decomposition product, in Tank 22 was investigated. Calculations show that Tank 22 would be ventilated with air at a rate sufficient to maintain a 1 -butanol concentration (volume \%) well below $25 \%$ of the lower flammability limit (LFL) for 1 butanol.

Tributylphosphate will not accumulate in Tanks 48,50, or 22 if added at concentrations up to $100 \mathrm{mg}$. Additionally, no significant quantities of TBP will be carried over to Tank 49 or further downstream. All soluble decomposition products are expected to be cycled from Tank 22 to Tank 48 and ultimately removed from the process in the decontaminated salt solution (DSS) waste stream. Since only minimal TBP and TBP decomposition products (e.g., dibutyl- and monobutylphosphate, 1-butanol, and phosphate) are expected to be carried over to Tank 49, there should be no effect on DWPF. Evaluation of material balance data in conjunction with uranium solubility experiments indicates no criticality problems will occur. Data indicate that ventilation of Tank 22 will prevent the TBP decomposition product, 1-butanol, from accumulating at a rate sufficient to produce a flammable concentration (at initial TBP feed concentrations of $100 \mathrm{mg} / \mathrm{L}$ ). Further calculation has determined that in the event of loss of ventilation in Tank 22, the time to reach LFL for 1-butanol would be 18.5 days.

\section{INTRODUCTION}

In-Tank Precipitation (ITP) is a process designed to remove soluble cesium and strontium from highly alkaline, salt solutions. 1 This is accomplished as a batch process in Tank 48 by precipitation and absorption using sodium tetraphenylborate (NaTPB) and monosodium titanate (MST). The cesium- and strontium-containing solids are concentrated to 10 weight percent by filtration. The resulting decontaminated salt solution filtrate, DSS, is processed through stripping columns to remove benzene formed by the radiolytic decomposition of NaTPB. The filtrate is stored temporarily in hold tanks and 
eventually is transferred to Tank 50 for processing into Saltstone. Second and third batches are processed in the same manner in the presence of the previous batch's concentrated solids. The accumulated cesium- and strontium-containing solids are washed and filtered to remove residual, soluble salts. The resulting spent wash water filtrate is also processed through the stripping columns to remove benzene and temporarily stored in hold tanks before eventually being sent to Tank 22 where it is mixed with spent wash water from the Late Wash Facility. The contents of Tank 22 are recycled to Tank 48 for use in the precipitation and concentration batch processes during the next ITP process cycle. The three precipitation and concentration batches and the washing process constitute one cycle.

The ITP benzene stripping columns have been observed to experience large pressure differentials across their structured packing. 2,3 The pressure differentials are the result of filtrate foaming within the columns. TBP (n-tributylphosphate) is an effective anti-foaming agent for the highly caustic salt solution filtrate and alleviates the pressure differential in the columns. 4,5 The Interim Waste Technology Section (IWTS) of the Savannah River Technology Center (SRTC) was requested (HLE-TTR-93040) 6 to evaluate potential adverse effects of TBP addition to the ITP process. Specifically, the concerns to be addressed included recycle of TBP throughout ITP, carryover to the Defense Waste Processing Facility (DWPF), and the disposal of TBP-containing decontaminated salt solution by Saltstone.

Several individual safety-related documents have been prepared which address differing aspects of TBP usage. 7-13 These documents were prepared by IWTS personnel and are related to TBP usage in Cold Chemical Testing. These documents are not necessarily applicable to normal ITP operation but are still relevant and may be informative. The documents describe chemical degradation of TBP, 7 consequences of addition of benzene stripper test solution to Tank $48,{ }^{8}$ allowable amounts of TBP in the ITP Feed and Hold Tanks, ${ }^{9}$ TBP vapor pressure, ${ }^{10}$ benzene and 1-butanol deflagration pressure calculations, ${ }^{11,12}$ and hydrogen generation from the radiolysis of TBP. 13

This document reports the results obtained from the investigation and evaluation of TBP recycle throughout ITP and its carryover to DWPF. A separate evaluation 14 of the disposal of TBP-containing decontaminated salt solution by Saltstone is being conducted by the Waste Disposal and Environmental Development Group of the IWTS. In addition, a materials of construction compatibility investigation is being conducted by the Equipment Engineering Section of the SRTC. ${ }^{15}$

\section{DISCUSSION}

To assess and evaluate the effect of TBP on ITP facilities, the flow path of TBP was determined and the expected concentration of TBP in the facilities was calculated. The flow path of TBP is readily identified. 1 However, several factors are capable of influencing TBP concentration. These factors include vanying facility volumes, solubility of TBP in the respective solutions and relatedly the degree of mixing or entrainment of insoluble materials in the solutions, hydrolysis of TBP, and radiolysis of TBP. Each of these factors are discussed in further detail later in this section. Based upon the maximum concentration of TBP expected in each facility, flammability, criticality, and DWPF-related issues are evaluated in this document.

\section{Tributylphosphate Flow Path and Recycle}

A schematic diagram of ITP is shown in Figure 1. The waste flow throughout the process is indicated on the diagram. As noted earlier, removal of soluble cesium and strontium from highly caustic, salt solutions is accomplished by precipitation and absorption in Tank 48.1 The cesium- and strontium-containing solids are concentrated to 10 weight percent by filtration and the resulting DSS filtrate is processed through stripping columns to remove benzene. The filtrate is stored temporarily in hold tanks and eventually is transferred to Tank 50 for processing into Saltstone. Second and thind batches are processed within Tank 48 in the presence of the first batch's concentrated solids. After three batches have been precipitated, the accumulated solids are washed and filtered to remove residual, soluble salts. The resulting spent wash water filtrate is also processed through the stripping columns to remove benzene and then temporarily stored in hold tanks. Eventually the spent wash water is sent to Tank 22 where it is mixed with 
spent wash water from the Late Wash Facility. The three precipitation and concentration batches and the washing process constitute one cycle. At this point, the 10 weight percent precipitate slurry contained in Tank 48 is transferred to Tank 49 for storage. Uttimately, the contents of Tank 49 will be processed through the Late Wash Facility and sent on to DWPF for processing. During the precipitate's processing in the Late Wash Facility, spent wash water will be generated and sent to Tank 22 and mixed with Tank 48 spent wash water. The contents of Tank 22 are eventually recycled for use in the precipitation and concentration batch processes during the next ITP process cycle within Tank 48.

Due to the large pressure differentials within the ITP benzene stripping columns, TBP will be added to the ITP process at a location downstream from the cross flow fitters (in the filtrate) but prior to the benzene stripping columns. During the ITP cycle, TBP will be added to both the DSS fittrate and the spent wash water filtrate. TBP added to the DSS filtrate during the concentration steps of the three precipitation batches will be transferred to Tank 50 and processed into Saltstone. There is a possibility that some residual TBP will remain in the filtrate hold tanks. The exact quantity is difficult to predict and will be a function of entrainment during transfer from the tanks. Tributylphosphate added to the spent wash water filtrate during the washing phase of the cycle will be sent to Tank 22. Since the contents of Tank 22 will be recycled to Tank 48 during the precipitation steps of the next cycle, TBP will also be recycled and thereby mixed with the contents of Tank 48. Depending upon the concentration, solubility, and rate of decomposition of TBP in Tanks 22 and 48, a mechanism exists whereby TBP could be sent to Tank 49 and ultimately to DWPF.

Figure 1. Schematic of the In-Tank Precipitation process facilities and waste flow path.

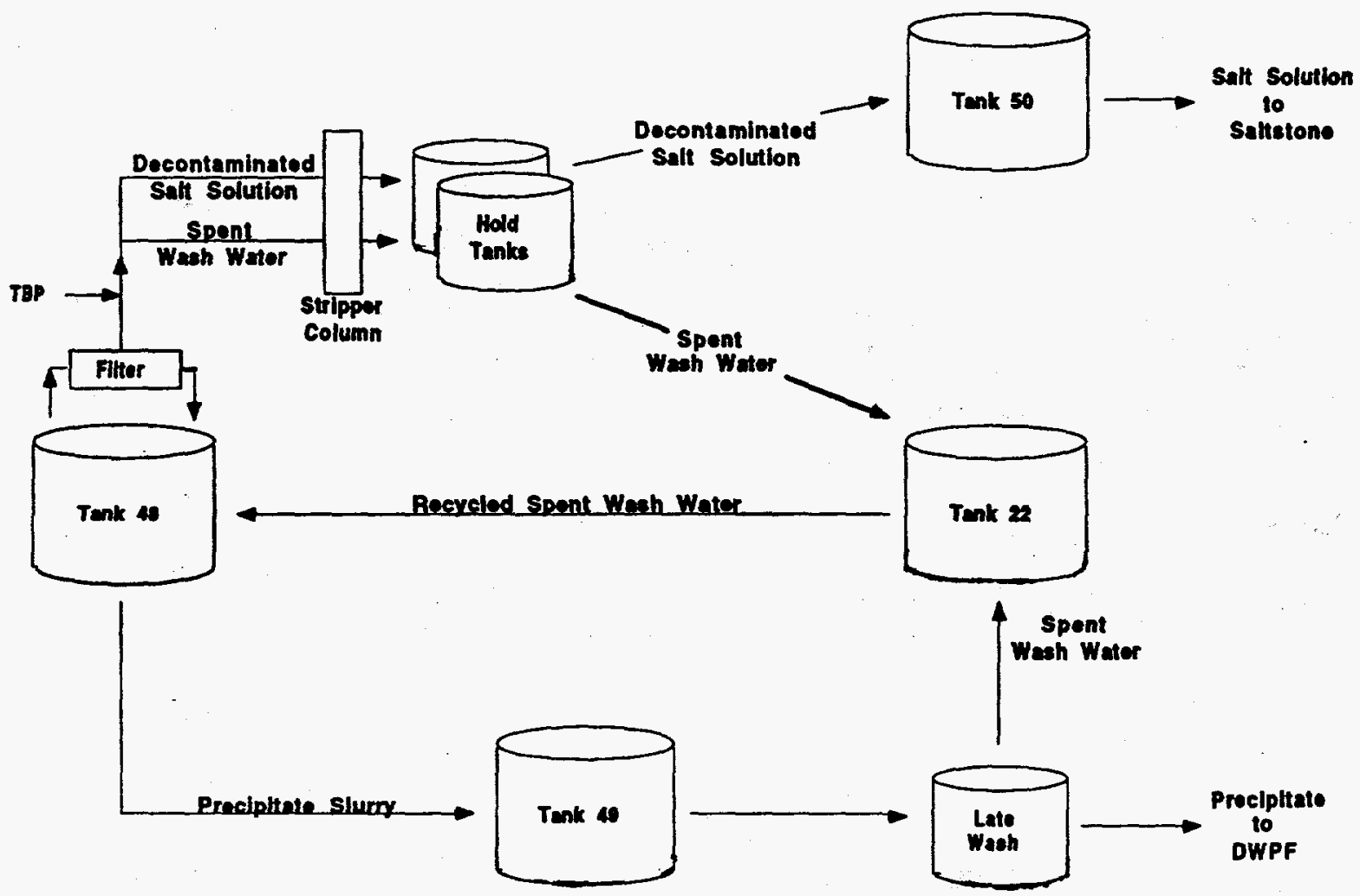

Material Balance

The effect of TBP upon ITP facilities is directly dependent upon the concentration of TBP within the respective facilities. There are several factors which can influence TBP concentration. The primary ones include varying facility volumes, solubility of TBP in the respective solutions, hydrolysis of TBP, and radiolysis of TBP. Each of these is further discussed in detail in the paragraphs that follow. 


\section{Solubillty of Trlbutylphosphate}

The solubility of TBP in DSS simulant was experimentally determined to be $1.1 \mathrm{mg} / \mathrm{L}$ at room temperature. 16 The solubility of TBP in wash water simulant was determined to be $16.0 \mathrm{mg} / \mathrm{L}$ at room temperature. ${ }^{16}$ Compositions of the simulated DSS and wash water are listed in Table $I$. As a check of the solubility test method, the solubility of TBP in water at room temperature was determined to be 385 $\mathrm{mg} / \mathrm{L} .{ }^{16}$ The solubility of TBP in water is reported in the literature to be $380 \mathrm{mg} / \mathrm{L}$ at $22^{\circ} \mathrm{C} .{ }^{17}$

Table I. Composition of DSS and washwater simulants.

Chemical
hydroxide
aluminate
nitrate
nitrite
carbonate
sulfate
oxalate
tetraphenylborate (saturated)

Total Sodium

DSS Simulant
(moles/liter)

1.48

0.31

1.74

0.67

0.16

0.14

0.015

0.002

\section{Wash Water Simulant (moles/liter)}
1.38
0.07
0.38
0.31
0.04
0.03
0.004
0.01
4.83
2.30

\section{Hydrolysis of Tributylphosphate}

Tributylphosphate hydrolyzes in the presence of strong alkaline solutions. 18 Products of the reaction are dibutylphosphate (DBP) and 1-butanol. Further hydrolysis of DBP results in the formation of monobutylphosphate (MBP) and 1-butanol. Hydrolysis of MBP yields phosphate and 1-butanol as reaction products. These reactions are shown below in equations 1a - 1d. The first order rate constant for hydrolysis of TBP at $30^{\circ} \mathrm{C}$ is reported as $0.007 \mathrm{hr}^{-1} .18$ Dibutylphosphate is reported to be considerably more resistant than TBP to alkaline hydrolysis. 18

$$
\begin{aligned}
& \left(\mathrm{CH}_{3} \mathrm{CH}_{2} \mathrm{CH}_{2} \mathrm{CH}_{2} \mathrm{O}_{3} \mathrm{PO}+\mathrm{OH}^{-} \rightarrow\left(\mathrm{CH}_{3} \mathrm{CH}_{2} \mathrm{CH}_{2} \mathrm{CH}_{2} \mathrm{O}_{2} \mathrm{PO}_{2}^{-}+\mathrm{CH}_{3} \mathrm{CH}_{2} \mathrm{CH}_{2} \mathrm{CH}_{2} \mathrm{OH}\right.\right. \\
& \left(\mathrm{CH}_{3} \mathrm{CH}_{2} \mathrm{CH}_{2} \mathrm{CH}_{2} \mathrm{O}_{2} \mathrm{PO}_{2}^{-}+\mathrm{OH}^{-} \rightarrow\left(\mathrm{CH}_{3} \mathrm{CH}_{2} \mathrm{CH}_{2} \mathrm{CH}_{2} \mathrm{O}\right) \mathrm{PO}_{3}^{-2}+\mathrm{CH}_{3} \mathrm{CH}_{2} \mathrm{CH}_{2} \mathrm{CH}_{2} \mathrm{OH}\right. \\
& \left(\mathrm{CH}_{3} \mathrm{CH}_{2} \mathrm{CH}_{2} \mathrm{CH}_{2} \mathrm{O}\right) \mathrm{PO}_{3}^{-2}+\mathrm{OH}^{-} \rightarrow \mathrm{PO}_{4}^{-3}+\mathrm{CH}_{3} \mathrm{CH}_{2} \mathrm{CH}_{2} \mathrm{CH}_{2} \mathrm{OH} \\
& \left(\mathrm{CH}_{3} \mathrm{CH}_{2} \mathrm{CH}_{2} \mathrm{CH}_{2} \mathrm{O}_{3} \mathrm{PO}+3 \mathrm{OH}^{-} \rightarrow \mathrm{PO}_{4}^{-3}+3 \mathrm{CH}_{3} \mathrm{CH}_{2} \mathrm{CH}_{2} \mathrm{CH}_{2} \mathrm{OH}\right.
\end{aligned}
$$

The hydrolysis of TBP in both DSS and wash water has not been accurately determined due to the low solubility of TBP in the salt solutions. ${ }^{19}$ Analysis of the experimental data gave hydrolysis rate constants for TBP which were approximately one onder of magnitude larger than that reported above. The. difference in the values may be associated with the degree of mixing and hydrolysis that may occur at the interface of the two solutions. Additionally, some experimental error was expected due to both the low total and soluble TBP concentrations being tested. Due to the low precision, the experimental rate constants are not considered valid absolute numbers. However, since the experimental rate constants are reasonably close to, but larger than, the referenced value listed above, they support the use of the referenced rate constant as a reasonable yet conservative value for calculating the material balance for 
TBP in ITP. For facilities containing solutions with TBP concentrations in excess of the solubility limit, a rate of hydrolysis was calculated assuming a steady state solubility limit concentration for TBP and the above listed rate constant (i.e. hydrolysis only occurs in the bulk aqueous phase and not at the interface of the two solutions).

For informational purposes, three graphs have been prepared based upon the literature rate for decomposition of TBP-containing solutions (Figures 2a, 2b and 2c). The graphs depict batch solutions in which no volume changes are occurring and where hydrolysis of soluble TBP is the only mechanism whereby TBP is removed. Figures $2 a$ and $2 b$ represent the hydrolysis of both $100 \mathrm{mg} / \mathrm{L}$ and $40 \mathrm{mg} / \mathrm{L}$ TBP-containing solutions. Both figures show the expected rate of decomposition for solutions with initial TBP concentrations greater than the TBP solubility limit. Figure $2 a$ shows the rate of decomposition for solutions with a solubility limit of $16.0 \mathrm{mg} /$ (simulating Tank 22 wash water). Figure $2 b$ shows the expected rate of decomposition for solutions with a TBP solubility limit of $1.1 \mathrm{mg} /$ (simulating Tank 50 DSS). The rate of decomposition is linear initially and is based upon maintaining the solubility limit concentration of TBP in the aqueous phase. Once enough TBP is decomposed to reduce the TBP concentration to below its solubility limit, TBP is decomposed logarithmically as predicted for a first order decomposition. This logarithmic decomposition is shown in expanded detail in Figure 2c. Figure $2 c$ represents the hydrolysis of a solution with an initial TBP concentration of $16.0 \mathrm{mg} / \mathrm{L}$ (again simulating Tank 22 wash water).

\section{Radiolysis of Tributylphosphate}

Information regarding the radiolysis of TBP in alkaline salt solutions has not been reported. However, radiolysis of pure TBP has been reported. 20-24 Radiolysis products (with their respective G-values) include dibutylphosphate (2.44 molecules/100 eV), monobutylphosphate (0.14 molecules/100 eV).

Figure 2a. Concentration of TBP based upon hydrolysis of both a $100 \mathrm{mgl}$ and $40 \mathrm{mg} / \mathrm{L}$ TBP. containing solution with a TBP solubility of $16.0 \mathrm{mg} /$. The graphs represent the expected rate of decomposition for Tank 22 wash water.

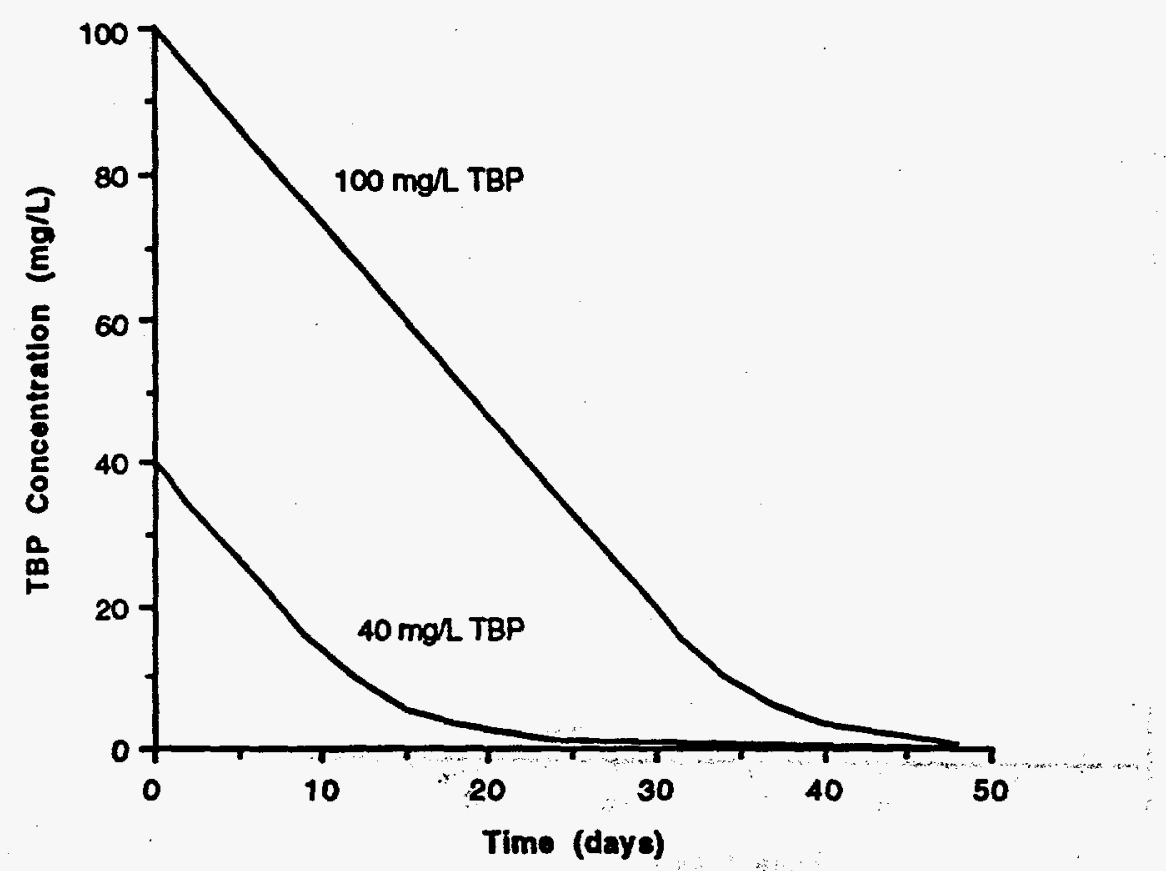


Figure 2b. Concentration of TBP based upon hydrolysis of both a $100 \mathrm{mg}$. and $40 \mathrm{mg}$. TBP. containing solution with a TBP solubility limit of $1.1 \mathrm{mg} /$. The graphs represent the expected rate of decomposition for Tank 50 DSS.

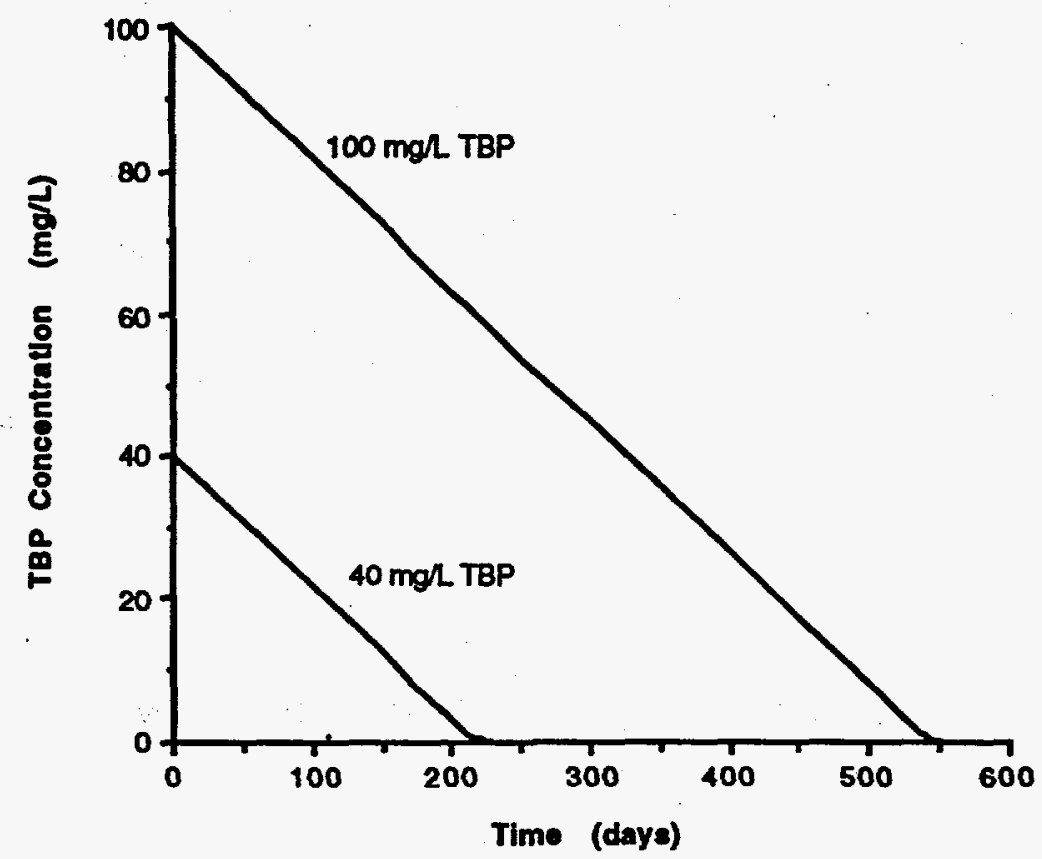

Figure 2c. Concentration of TBP based upon hydrolysis of a solution with an initial TBP concentration equal to the TBP solubility limit (16.0 mg $\mathrm{L}$ simulating Tank 22 wash water).

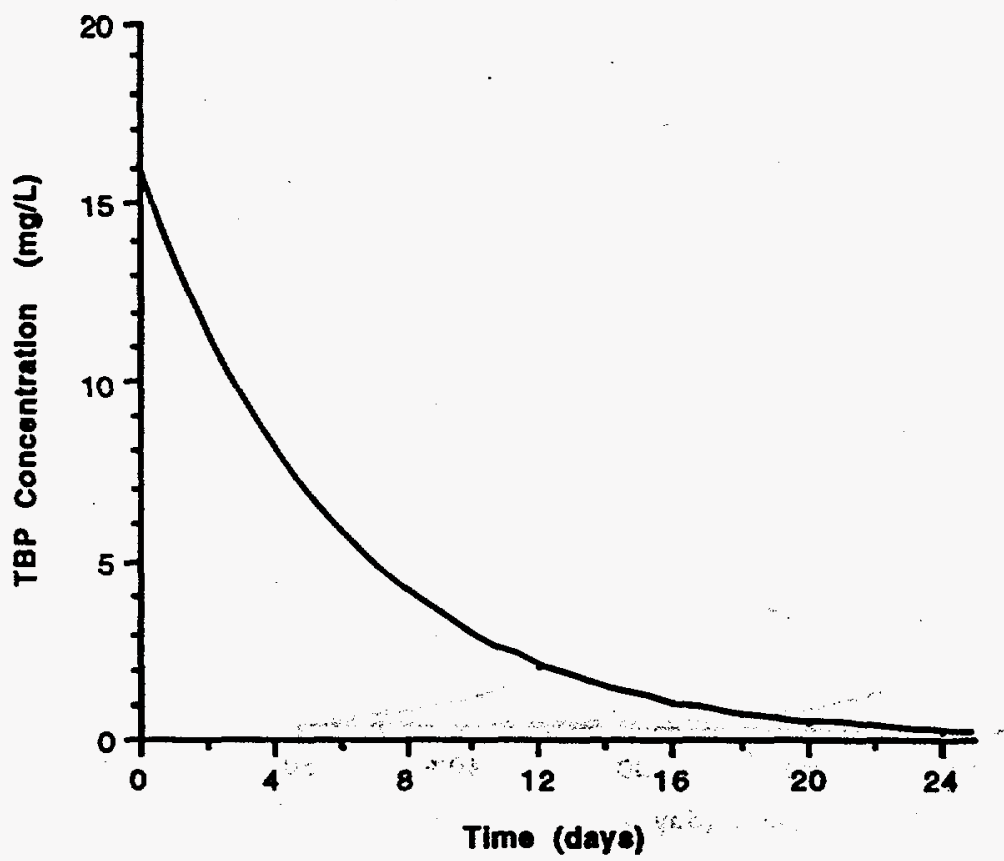


hydrogen ( 1.73 molecules/100 eV), and various 3 and 4 carbon atom decomposition compounds including 1-butanol, butane, propane, etc. (cumulative value for all of these species is 0.65 molecules/100 eV).

\section{Material Balance Calculations}

A material balance was performed for the addition of TBP to the ITP facilities. The material balance was calculated for the addition of two TBP concentrations (40 mg/L and $100 \mathrm{mg} / \mathrm{L}$ ). In each case, the TBP material balance was based upon the ITP flow sheet representing the ITP process with late wash. ${ }^{1}$ The TBP material balance was determined for a period of three cycles. The data obtained for cycle 2 and cycle 3 were identical, indicating that there was no "roll-over" effect (i.e., no TBP was accumulating in any facility from one cycle to the next). The material balance data are contained in Appendices $A$ and $B$ at the end of this document. The only facilities factored into the material balance are Tanks 48, 22, and 50. The hold tanks and Tank 49 were not considered since no dilution or concentration activities occur within these facilities. There is a possibility that some residual TBP will remain in the filtrate hold tanks. The exact quantity is difficult to predict and will be a function of entrainment during transfer from the tanks. Retention of residual TBP in the hold tanks was not accounted for in this material balance due to the difficulty involved in predicting actual amounts involved.

The material balance was calculated using the above information regarding solubility and hydrolysis. Furthermore, a few conservative yet reasonable assumptions were made in order to obtain the material balance. These assumptions included the following:

- Late Wash spent wash water is sent to Tank 22 at the average rate of of 2809 gallons per day. $t$ is assumed that this spent late wash water is similar in composition to Tank 48 spent wash water and will not change TBP solubility in Tank 22 significantly.

- TBP is not introduced into Tank 48 until the second cycle since the contents of Tank 22 used in the precipitation steps of cycle 1 do not contain TBP. TBP is not introduced into Tank 22 until the washing stage of cycle 1.

- TBP quantities (both decomposed and remaining) are calculated for the end of each step and are based upon the remaining TBP concentration data from the step immediately preceding it.

- Only soluble TBP will be recycled from Tank 22 to Tank 48 since any insoluble TBP would remain in a layer on the surface of the wash water in Tank 22. For information purposes, if all of the TBP (assuming $100 \mathrm{mg} / \mathrm{L}$ case) transferred to Tank 22 during one cycle were collected as a layer on the surface, it would be less than 0.010 inch thick.

- Hydrolysis of TBP in Tank 22 is not diffusion controlled (i.e., a TBP solubility limit concentration is maintained in the aqueous phase when TBP is present above the solubility limit).

- The only TBP degradation product produced, which is expected to be soluble to any appreciable degree, is 1-butanol. 25 The recycle of the other degradation products (dibutylphosphate and monobutylphosphate) is expected to be similar to that of TBP and should be minimal.26,27

- Radiolytic decomposition of TBP in Tank 48 was not accounted for since very little TBP was recycled to Tank $\mathbf{4 8}$ and the amount which is recycled is rapidly decomposed and removed by filtration and washing.

- Decomposition of TBP within Tank 50 was not calculated. The actual concentration of TBP in Tank 50 will be less than the TBP addition concentration due to the addition of ETF evaporator concentrate to the DSS filtrate. This will further serve to dilute the TBP. Decomposition of TBP in Tank 50 will be slow (relative to Tank 22) due to the low solubility of TBP in DSS (Figure 2a vs. 2b). The effect of TBP in Saltstone is the subject of another document. 14 
The material balances yield the same observations for both $40 \mathrm{mg} /$ and $100 \mathrm{mg} / \mathrm{TBP}$ addition concentrations. A summary of these observations follows:

- Tributylphosphate is not accumulated from one cycle to the next cycle.

- $99 \%$ of the TBP added to Tank 22 is decomposed within the tank.

- Only small amounts of TBP (<1 mg/L) will be recycled from Tank 22 into Tank 48.

- All TBP which is recycled from Tank 22 to Tank 48 is eventually decomposed or filtered off.

Data contained in the TBP material balances in Appendices A and B have been summarized in Table II below. Only the most important and pertinent information is provided due to the large quantity of data generated. In addition to the table, a graph representing the TBP concentration in Tank 22 was prepared using the material balance TBP concentration data contained in Appendices A and B. The graph represents a time period of three cycles in the ITP process (the data for the first cycle represents initial start-up). As can be seen in Figure 3, the maximum TBP concentration is less than $15.4 \mathrm{mg} / \mathrm{L}$ and 6.2 $\mathrm{mg} / \mathrm{L}$ for the $100 \mathrm{mg} / \mathrm{L}$ and $40 \mathrm{mg} / \mathrm{TBP}$ feed rates, respectively.

Table II. Summary of TBP material balance data contained in Appendices A and B. The material balance data were prepared for TBP additions at $40 \mathrm{mg} / \mathrm{L}$ and $100 \mathrm{mg} / \mathrm{L}$.

TBP Addition Concentration

Tank Feature

Tank 22 (per cycle)

maximum TBP. Concentration:

Amount of TBP decomposed per cycle:

Tank 48 (per cycle)

maximum TBP Concentration:

amount of TBP recycled to (from Tank 22):

TBP transferred to Tank 49

Tank 50 (per batch)

maximum amount of TBP:

TBP Addition Concentration

$40 \mathrm{mon}$

$100 \mathrm{mo} / \mathrm{h}$

$6.2 \mathrm{mg} / \mathrm{L}$

$49.9 \mathrm{Kg}$

$0.2 \mathrm{mg} / \mathrm{L}$

$743 \mathrm{~g}$

$0 \mathrm{~g}$

$127 \mathrm{Kg}$
$15.4 \mathrm{mg} / \mathrm{L}$

$125 \mathrm{Kg}$

$0.6 \mathrm{mg} / \mathrm{L}$ $1860 \mathrm{~g}$

$0 \mathrm{~g}$

$317 \mathrm{Kg}$

\section{Criticality Issues - The Effect of TBP on Solubility of Uranium and Loading onto MST}

A review of possible impacts of TBP and TBP hydrolysis products (i.e., DBP and MBP) on nuclear criticality safety in the ITP process identified the following: (1) increase solubility of uranium, (2) increase loading of uranium onto MST, and (3) accumulation of fissile material in a separate layer or TBP residue in the Filtrate Hold Tanks or Tank 22. Since TBP and DBP are known to be complexants for metal ions, the addition of TBP could alter the distribution of fissile material in the ITP process. For example, addition of TBP could increase the solubility of uranium resulting in a high chemical potential for loading onto MST. Although data in dilute caustic/carbonate solution suggest that the solubility would not be increased, there was no definitive data at higher salt solutions.

A set of tests were conducted to determine the effect of adding $50-500 \mathrm{ppm}$ of tributylphosphate on the solubility of uranium in a concentrated salt solution. The salt solution test had a $\left[\mathrm{Na}^{+}\right]$of 4.3 molar and was comprised of the following salts; sodium hydroxide $(2.0 \mathrm{M})$, sodium nitrate $(1.0 \mathrm{M})$, sodium nitrite $(0.1 \mathrm{M})$, sodium aluminate $(0.33 \mathrm{M})$, sodium sulfate $(0.40 \mathrm{M})$, and sodium carbonate $(0.02 \mathrm{M})$. This solution was 
Figure 3. Concentration of TBP in Tank 22 over three ITP process cycles.

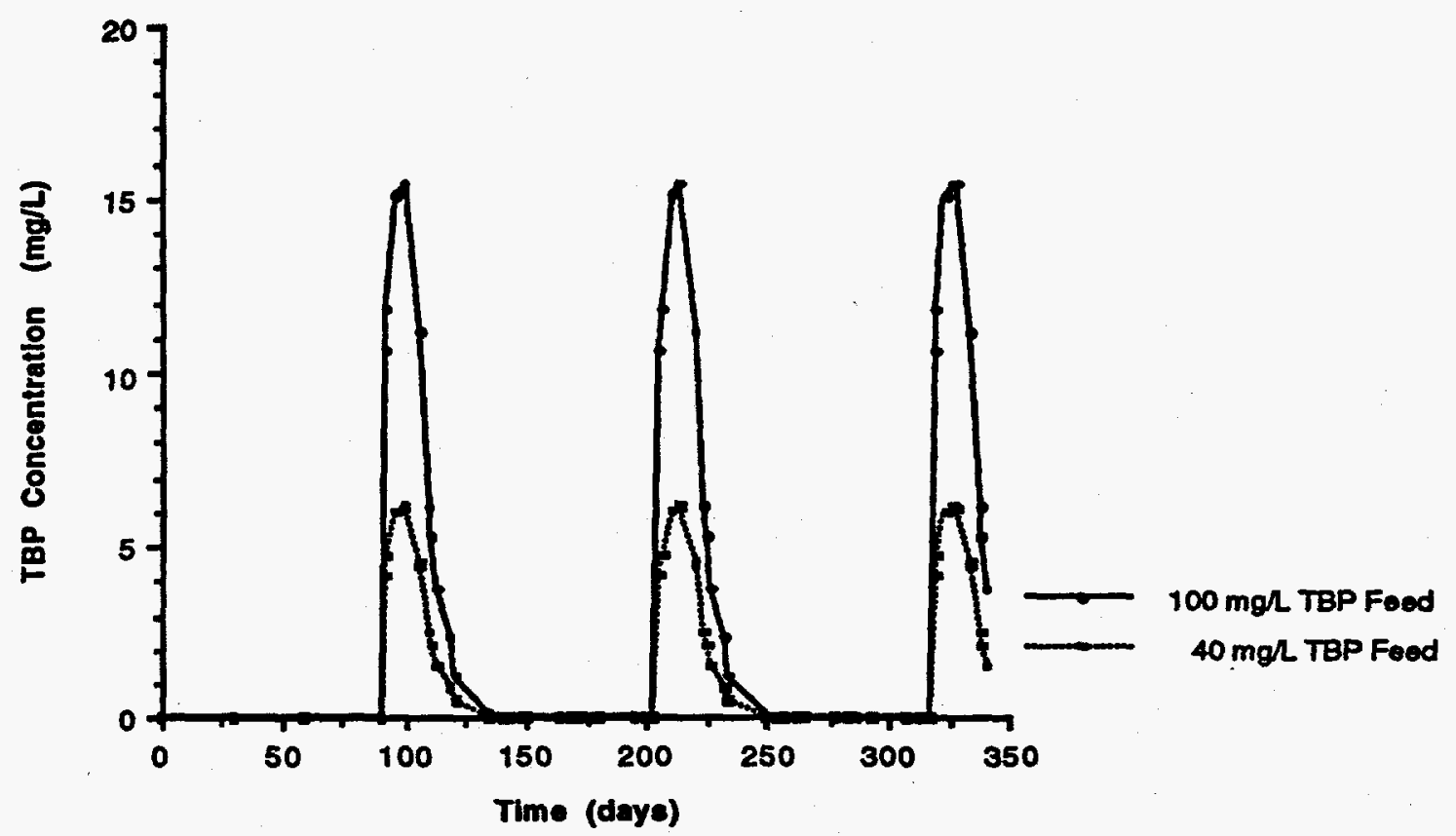

previously selected for use in batch loading tests with monosodium titanate (MST) because of its high solubility for uranium and plutonium relative to the range of salt solution compositions which will be processed in ITP. 28

Analytical results are presented in Table III. The results indicated that the presence of 50.9 and 508 ppm (w/w) of TBP does not affect the solubility of uranium in concentrated salt solution. Thus, the addition of TBP would not be expected to increase the amount of uranium released to saltstone through ITP. The decreasing solubility with time is attributed to changes in the phase of the uranium and the composition of the salt solution 29 (i.e. with time the form of uranium is changing to a less soluble form, and the salt solution composition also is changing such that it decreases the solubility of any particular uranium phase). It is also possible that the undissolved and/or precipitated uranium is dissolving in an excess TBP phase. However, if uranium were dissolving into an excess TBP phase, it would be expected that the rate of change in the uranium concentration would be different for the bottles with TBP than that without TBP. The uranium dissolved in the TBP phase would provide an additional source of uranium for dissolution into the aqueous phase. This should alter the rate of decrease in uranium concentration. Since this was not observed, it is concluded that the uranium is not dissolving in an excess TBP phase.

TBP is a neutral molecule, and therefore, should not exhibit a significant tendency to adsorb onto the MST. If adsorption would occur, it will be through the polar phosphate end of the molecule. Since this is also the portion which complexes with uranium to form a more soluble compound than the uncomplexed uranium form, the loading of uranium onto the MST cannot be increased because the uranium complexing sites are tied up with the MST (probably by hydrogen bonding). Therefore, it is concluded that the use of TBP in ITP as a defoaming agent will not impact the solubility of uranium or the loading of uranium onto the monosodium titanate.

\section{Flammability lssues}

Flammability of TBP in any of the process tanks is of no concern due to its extremely low vapor pressure. 10 The flashpoint of pure TBP is $193^{\circ} \mathrm{C}$. However, the flammability of TBP degradation products is of 
Table III. Uranium Concentrations of Test Solutions.

Average Uranium Concentration (mo/L)

\begin{tabular}{cc} 
Elapsed Time (days) & No TBPb \\
\hline 1 & $12.4 \pm 0.21$ \\
2 & $10.8 \pm 0.071$ \\
7 & $7.81 \pm 0.40$ \\
14 & $6.89 \pm 0.21$ \\
28 & $5.99 \pm 0.40$
\end{tabular}

\section{9pem TBPC}

$13.0 \pm 0.17$

$11.0 \pm 0.43$

$8.32 \pm 0.19$

$6.98 \pm 0.15$

$5.95 \pm 0.20$
508 pom TBPC

$12.8 \pm 0.25$

$10.8 \pm 0.26$

$8.44 \pm 0.28$

$7.01 \pm 0.18$

$6.18 \pm 0.25$

aUranium concentrations were determined by kinetic phosphorescence spectroscopy.

bAverage and standard deviation of duplicate measurements.

CAverage and standard deviation of duplicate measurements for replicate tests.

concem. In particular, the two degradation products which were investigated are hydrogen and 1-butanol. Hydrogen is formed from the radiolysis of TBP. Previous calculations showed that a TBP concentration of $4000 \mathrm{mg} / \mathrm{L}$ was necessary in Tank 48 in order to generate a $1.0 \%$ increase in hydrogen formation. ${ }^{13}$ Given that the TBP concentration in Tank 48 is expected to be less than $1 \mathrm{mg} /$, hydrogen formation from the radiolysis of TBP does not pose any flammability concems. Radiolysis of TBP in the other facilities (e.g., Tank 22) is of little concem due to both the low TBP concentration and the low levels of radioactivity.

Tank 22 will contain the largest quantity of TBP and TBP decomposition products and therefore is of greatest concern for 1-butanol flammability. Calculations based upon the addition of $100 \mathrm{mg} / \mathrm{LBP}$ show that $125 \mathrm{~kg}$ of TBP are decomposed in Tank 22 during one process cycle. Additional calculations based upon this amount in conjunction with the rate of hydrolysis of TBP have been performed. These calculations are presented in Appendix $\mathbf{C}$ and indicate that under worst case conditions (as listed in Appendix C), ventilation of Tank 22 would prevent the TBP decomposition product, 1-butanol, from accumulating at a rate sufficient to produce a flammable concentration. The data indicate that under these conditions, 1-butanol would be produced at a volume $\%$ concentration of $0.015 \%$. The LFL for 1-butanol is $1.45 \% .30$ Current ITP OSR limits are set at $25 \%$ of the composite LFL for Tank 22.31 Further calculation has determined that in the event of loss of ventilation in Tank 22, the time to reach LFL for 1-butanol would be 18.5 days.

Flammability of 1-butanol in Tanks 48 and 49 will be less likely for a few reasons: (1) less TBP and TBP decomposition products are present, (2) both tanks are inerted with nitrogen, and (3) both tanks will be ventilated with nitrogen at a rate equivalent to that of Tank 22 with air. Once design is completed, the TBP injection system should be more adequately investigated with regards to flammability issues. Also, the potential for flammability in the fittrate hold tanks should be more thoroughly investigated once a more accurate determination is made regarding the amount of residual TBP that will remain in the filtrate hold tanks. The filtrate hold tanks are maintained with a maximum oxygen content (MOC) of $3.6 \%$. The MOC for 1 -butanol is not readily available.

\section{DWPF Issues}

The material balance described previously indicates that no TBP will remain further downstream than Tank 48. Therefore, TBP itself should have no direct impact on DWPF facilities. Furthermore, any soluble (or insoluble) TBP degradation products (e.9., phosphate, 1-butanol, etc.) which will be transported from Tank 22 into Tank 48 (exact quantities will depend upon their respective solubilities) should eventually be removed from Tank 48 in the DSS filtrate during the concentration steps. Any additional residual 
degradation products will be removed during the washing portion of the ITP cycle. Therefore, only extremely small amounts of TBP and TBP degradation products will likely be transported to Tank 49. Again, processing of the precipitate slumy in the Late Wash facility would further serve to remove and reduce (by approximately a factor of 10) the TBP and TBP degradation products in the unlikely event that any of the materials were still present.

In addition to the material balance, experiments have been conducted by the Defense Waste Processing Technology Section (DWPT) of the SRTC examining the effects of using TBP as a defoaming agent in DWPF. ${ }^{32}$ Results indicate that TBP is ineffective in controlling foam. For informational purposes, there would be some concem associated with formation of a red-oil in the SRAT/SME if excess amounts of nitric acid were to be added and if TBP were to be present that far into the process. However, this should not be a problem since TBP is not expected to be present downstream from Tank 48.

\section{CONCLUSION}

Based upon a TBP addition concentration of $100 \mathrm{mg} / \mathrm{L}$ or less, the material balance data and all relevant information indicate that TBP will not accurmulate in Tanks 22, 48, or 50. Furthermore, no TBP will be carried over to Tank 49 or further downstream. All soluble decomposition products are expected to be recycled from Tank 22 to Tank 48 and ultimately removed from the process in the DSS waste stream. Since no TBP or TBP decomposition products (i.e., DBP, MBP, 1-butanol, and phosphate) are expected to be recycled to Tank 49, there should be no effect on DWPF. Evaluation of the material balance data in conjunction with uranium solubility experiments indicates no criticality problems will occur. Effects of decomposition products were also considered. Flammability of 1-butanol in Tank 22 was investigated. It was determined that Tank 22 would be ventilated with air at a rate sufficient to maintain a 1-butanol concentration (volume \%) well below $25 \%$ of the LFL for 1-butanol (based upon a TBP feed addition of $100 \mathrm{mg} / \mathrm{L}$ ). Further calculation determined that in the event of loss of ventilation in Tank 22, the time to reach LFL for 1-butanol would be 18.5 days.

\section{QUALITY ASSURANCE}

All work was conducted in accordance with the SRL Quality Assurance Program. Experimental work was performed in accordance with Task Technical Plans WSRC-RP-93-766 "Salt Solution Foaming Characteristics Technical Task Plan" and WSRC-RP-93-1010 "Task Technical Plan to Evaluate the Solubility of Tributylphosphate in ITP Salt Solutions". Salt solutions were prepared from reagent grade chemicals. Chemical analyses were performed by the Analytical Development Section of SRTC.

\section{REFERENCES}

1. D. D. Walker, "Material Balance for the ITP Process with Late Washing," SRT-LWP-92-074, July 14, 1992.

2. M. J. Barnes, "Summary of Meeting to Discuss Potential Foaming Problems in the ITP Stripper (U)," SRT-LWP-93-019, March 2, 1993.

3. G. K. Georgeton, "Pilot Scale Stripping Column Test Program (U)," WER-HLE-930653, March 16, 1993.

4. M. J. Barnes, "Lab-Scale Defoaming Tests on the In-Tank Precipitation Benzene Stripper Columns (U)," SRT-LWP-93-021, March 19, 1993.

5. J. F.McGlynn, "Evaluating Defoaming Agents for the Stripping Columns at the In-Tank Precipitation Facility (U)," WSRC-RP-93-928, June 30, 1993.

6. Task Technical Request \# HLE-TTR-93040.

7. M. J. Bames, "Chemical Degradation of TBP (U)," SRT-LWP-93-044, May 26, 1993. 
REFERENCES (continued)

8. J. P. Bibler, "Calculations Concerning and Consequences of Addition of Stripper Test Solution to Tank 48 (U)," SRT-LWP-93-026, March 30, 1993.

9. M. J. Bames, "Allowable TBP in Feed, Hold Tanks (U)," SRT-LWP-93-030, May 17, 1993.

10. M. J. Bames, "Tributylphosphate Vapor Pressure (U)," SRT-LWP-93-045, June 15, 1993.

11. D. D. Walker, "Consequences of a Stripper Building Stack Deflagration," IWT-LWP-90-0177, September 24, 1990.

12. M. J. Bames, "1-Butanol Deflagration Pressures and LFL Calculations," SRT-LWP-93-043, May 21, 1993.

13. M. J. Bames, "Hydrogen Generation from the Radiolysis of Tributylphosphate (U)," SRT-LWP-93-054, July 22, 1993.

14. C. A. Langton and E. G. Orebaugh, "Evaluation of the Effects of Tributylphosphate (Defoaming Agent) in Z-Area Saltstone (U)," WSRC-RP-93-1185, August 18, 1993.

15. T. E. Skidmore, "ITP Materials Compatability Issues (U)," SRT-MTS-93-5149, August 20, 1993.

16. R. F. Swingle, Unpublished results, WSRC-NB-93-260, pp. 7-20, August 4, 1993.

17. W.W. Schulz and J. D. Navratil, Science and Technology of Tributyl Phosphate: Volume 1 Synthesis, Properties, Reactions and Analysis, CRC Press, Inc., Boca Raton, Florida, (1984), pp. 32-33.

18. Ibid, pp. 149-151.

19. R. F. Swingle, "Calculated Rate Constants for Hydrolysis of Tributylphosphate in Simulated Decontaminated Salt Solution (U)," SRT-LWP-93-076, October 26, 1993.

20. R. W. Wilkinson and T. F. Williams, J. Chem Soc., 4098 (1961).

21. R. M. Wagner, E. M. Kinderman, and L. H. Towle, Ind. Eng. Chem., 51,45 (1959).

22. J. G. Burr, Radiation Research, 8 , 214 (1958).

23. L. L. Burger and E. D. McClanahan, Ind. Eng. Chem., 50, 153 (1958).

24. R. W. Wilkinson and T. F. Williams, Nature, 179, 540 (1957).

25. Material Safety Data Sheet, J. T. Baker Chemical Co., Phillipsburg, New Jersey.

26. Material Safety Data Sheet, Eastman Kodak Company, Rochester, New York

27. Material Safety Data Sheet, Pfaltz \& Bauer, Inc., Waterbury, Connecticut.

28. D. T. Hobbs and S. D. Fleischman, "Fissile Solubility and Monosodium Titanate Loading Tests," WSRC-RP-92-1273, February 12; 1993.

29. D. T. Hobbs and T. B. Edwards, "Solubility of Uranium in Alkaline Salt Solutions," WSRC-TR-93-454, draft document, August 27, 1993. 
S. D. Fink

13

WSRC-RP-93-1162

REFERENCES (continued)

30. R. C. Weast, CRC Handbook of Chemistry and Physics, 60th edition, CRC Press, Inc., Bock Ration, Florida, (1980), p. D-122.

31. "Occupational Safety Requirements for the In-Tank Precipitation Process at the Savannah River Site (U)," WSRC-RP-90-1124 Rev. 2, June 18, 1993.

32. L. F. Landon, "Antifoam Scouting Tests," Inter-office Memorandum, July 29, 1993.

33. E. W. Washbum, International Critical Tables, Volume III, 1st edition, McGraw Hill Book Company, New York, New York, (1928), p. 388.

34. "Additional Analysis for DWPF Feed Preparation by In-Tank Processing (U),." DPSTA-200-10, Sup. 18, Addendum 1, Draft Rev. 1.

Technical review performed by:

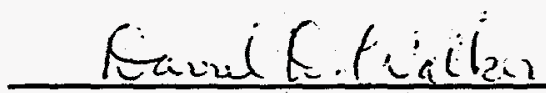

D. D. Walker, Bldg. 773-A

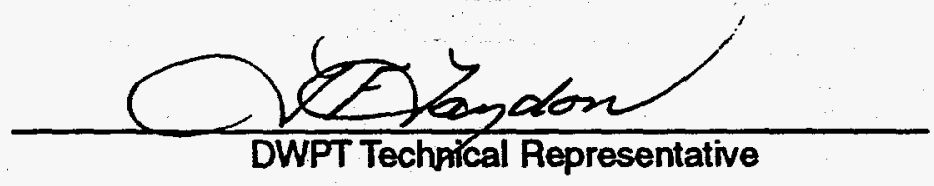


APPENDIX A

The material balance for the addition of $40 \mathrm{mgh}$ TBP in Tanks 48,50 , and 22 is shown below.

\section{Concentration of TBP Added: \\ TPB Solublity in DSS: \\ TPB Solubllity in Wash Water: \\ DSS TBP Hydrolyels Rate: \\ TBP Hydrolysis Rate Constant:}

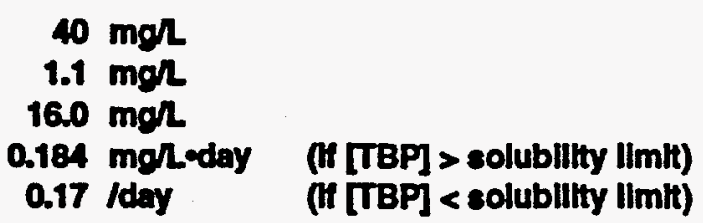

\begin{tabular}{|c|c|c|c|c|c|c|c|}
\hline \multicolumn{3}{|c|}{ Procese } & \multicolumn{5}{|c|}{ Tank 48-Cycle 1} \\
\hline Step & 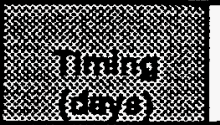 & $\begin{array}{l}\text { Volume } \\
\text { Change } \\
\text { (Kgal) }\end{array}$ & 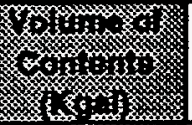 & $\begin{array}{c}\text { TBP } \\
\text { Decomp } \\
\text { (g) }\end{array}$ & 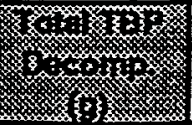 & $\begin{array}{c}\text { Remaining } \\
\text { TBP } \\
\text { (g) }\end{array}$ & 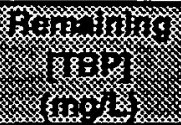 \\
\hline & & & 貿 & & & & \\
\hline \multicolumn{8}{|l|}{ Batch 1} \\
\hline Transfer SS & 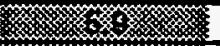 & 630 & $x \leqslant$ & 0 & x & 0 & 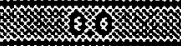 \\
\hline Transfer AWW & 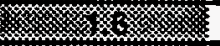 & 217 & 糸 & 0 & 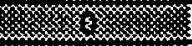 & 0 & 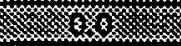 \\
\hline Add Misc. Soln. & x & 40.1 & 驾 & 0 & $\xi$ & 0 & m \\
\hline Filter to 7.5 wt $\%$ & 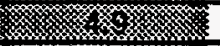 & 813.5 & x & 0 & s & 0 & stis \\
\hline Filter to 10 wt \% & sin & 18.4 & 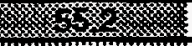 & 0 & 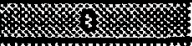 & 0 & 3 \\
\hline Stop/Start & 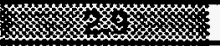 & - & 贺 & 0 & 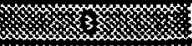 & 0 & 8 \\
\hline & & & & & 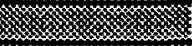 & & \\
\hline \multicolumn{8}{|l|}{ Batch \# 2} \\
\hline Transfer SS & 站 & 630 & 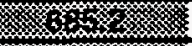 & 0 & 勞箘 & 0 & 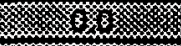 \\
\hline Transfer AWW & 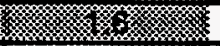 & 217 & x & 0 & 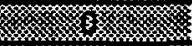 & 0 & m \\
\hline Add Misc. Soln. & s & 27 & $\sin$ & 0 & s. & 0 & s \\
\hline Filter to 7.5 wt $\%$ & 悲 & 806.7 & m & 0 & 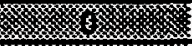 & 0 & sctis \\
\hline Filter to $10 \mathrm{wt} \%$ & 桨 & 30.6 & w & 0 & 玹 & 0 & 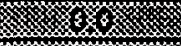 \\
\hline \multirow[t]{2}{*}{ Stop/Start } & 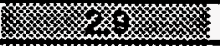 & - & ) & 0 & 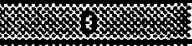 & 0 & 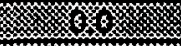 \\
\hline & 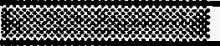 & & xy & & 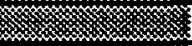 & & $x$ \\
\hline \multicolumn{8}{|l|}{ Batch : 3} \\
\hline Transfer SS & sis & 630 & sy & 0 & 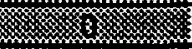 & 0 & s. \\
\hline Transfer AWW & 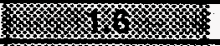 & 217 & 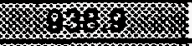 & 0 & 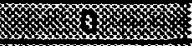 & 0 & shes \\
\hline Add Misc. Soln. & X & 27 & 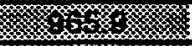 & 0 & 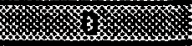 & 0 & s \\
\hline Filter to 7.5 wt $\%$ & s. & 798.6 & stis & 0 & 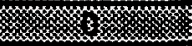 & 0 & 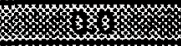 \\
\hline Fitter to $10 w t \%$ & 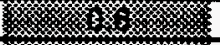 & 40.2 & 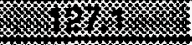 & 0 & 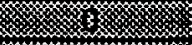 & 0 & 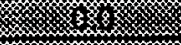 \\
\hline Stop/Start & 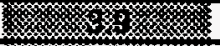 & - & x & 0 & 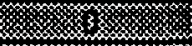 & 0 & 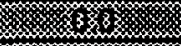 \\
\hline \multicolumn{8}{|l|}{ Wash Cycle } \\
\hline Wash & x & 50 & 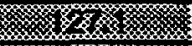 & 0 & 3 & 0 & s.ts \\
\hline Add $\mathrm{NaOH}$ & 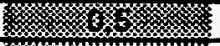 & 8 & 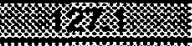 & 0 & 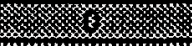 & 0 & 㫨 \\
\hline Wash & 椊 & 70 & 好 & 0 & 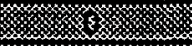 & 0 & 5 \\
\hline Add $\mathrm{NaOH}$ & $x$ & 6 & 絔 & 0 & 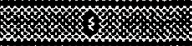 & 0 & s. \\
\hline Wash & 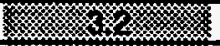 & 65 & 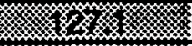 & 0 & 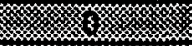 & 0 & 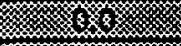 \\
\hline Add NaNO2 & 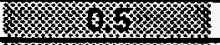 & 8 & 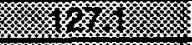 & 0 & 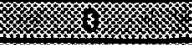 & 0 & 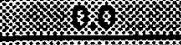 \\
\hline Wash & 嬨 & 125 & 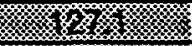 & 0 & 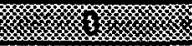 & 0 & s. \\
\hline Sample & 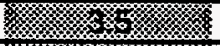 & - & 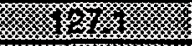 & 0 & 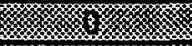 & 0 & tos \\
\hline Transfer Slumy & m & 130 & 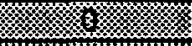 & 0 & 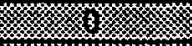 & 0 & 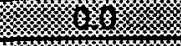 \\
\hline Stop & 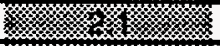 & - & 烈 & 0 & 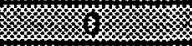 & 0 & 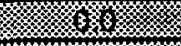 \\
\hline & 穷 & & 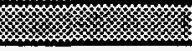 & & & & \\
\hline
\end{tabular}

SS = Salt Solution

RWW = Recycled Wash Water

Halicized volume changes indicate removal from Tank 48.

Non-italicized volume changes indicate additions to Tank 48.

The wash cycle volume changes do not cause volume level changes in Tank 48 since the washwater and inhibitor solutions are added and removed simultaneously.

The volume of the contents in Tank 22 is incrementally increased by Late washwater at the rate of 2809 gallons per day. 
APPENDIX A (continued)

Concentration of TBP Added: TPB Solubilty in DSS:

TPB Solubillty in Wach Water:

RWW TBP Hydrolysls Rate:

TBP Hydrolyels Rate Constant:

\author{
$40 \mathrm{mgh}$ \\ $1.1 \mathrm{mgh}$ \\ $16.0 \mathrm{mgh}$ \\ 0.17 /day
}

$2.670 \mathrm{mg}$ Leday (if [TBP] > solubllity limit) (if [TBP] < colubllity IImit)

\begin{tabular}{|c|c|c|c|c|c|c|c|}
\hline \multicolumn{3}{|c|}{ Process } & \multicolumn{5}{|c|}{ Tank 22-Cycle 1} \\
\hline Step & 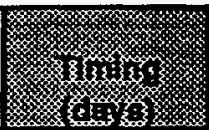 & $\begin{array}{l}\text { Volume } \\
\text { Change } \\
\text { (Koal) }\end{array}$ & 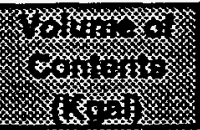 & $\begin{array}{c}\text { TRP } \\
\text { Dacomp } \\
\text { (q) }\end{array}$ & 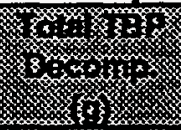 & $\begin{array}{c}\text { Remaining } \\
\text { TBP } \\
\text { (g) }\end{array}$ & 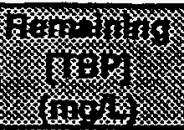 \\
\hline \multirow{2}{*}{\multicolumn{8}{|c|}{ Batch 1}} \\
\hline & & & & & & & \\
\hline Transfer SS & s & 630 & 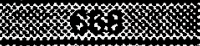 & 0 & 䇾 & 0 & 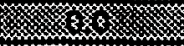 \\
\hline Transfer RWW & x & 217 & 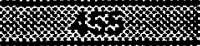 & 0 & x & 0 & s. \\
\hline Add Misc. Soln. & 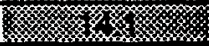 & 40.1 & 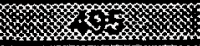 & 0 & 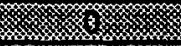 & 0 & s. \\
\hline Filter to $7.5 w t \%$ & 帘 & 813.5 & s.t. & 0 & K & 0 & sis \\
\hline Filter to $10 \mathrm{wt} \%$ & 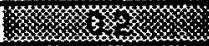 & 18.4 & $x$ & 0 & 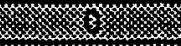 & 0 & 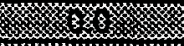 \\
\hline Stop/Start & 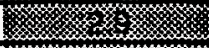 & - & 柇 & 0 & 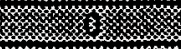 & 0 & 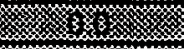 \\
\hline \multicolumn{8}{|l|}{ Batch \# 2} \\
\hline Transfer SS & sin & 630 & sts & 0 & 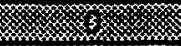 & 0 & 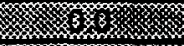 \\
\hline Transfer AWW & 聯 & 217 & sis & 0 & (x) & 0 & 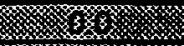 \\
\hline Add Misc. Soln. & 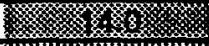 & 27 & sing & 0 & 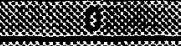 & 0 & 2 \\
\hline Filter to 7.5 wt $\%$ & 管 & 806.7 & w & 0 & K & 0 & 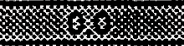 \\
\hline Filter to $10 \mathrm{wt} \%$ & 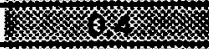 & 30.6 & 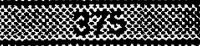 & 0 & s. & 0 & 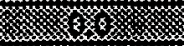 \\
\hline Stop/Stant & rets & - & 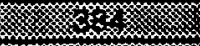 & 0 & 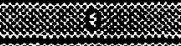 & 0 & 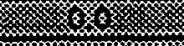 \\
\hline \multicolumn{8}{|l|}{ Batch 3} \\
\hline Transfer SS & 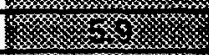 & 630 & 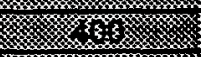 & 0 & 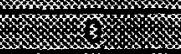 & 0 & 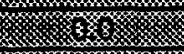 \\
\hline Transfer RWW & m & 217 & sistis & 0 & 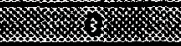 & 0 & 列 \\
\hline Add Misc. Soln. & 浆好 & 27 & 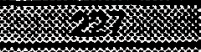 & 0 & 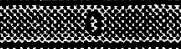 & 0 & 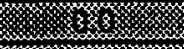 \\
\hline Filter to 7.5 wt \% & $x$ & 798.6 & 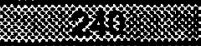 & 0 & 准 & 0 & 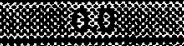 \\
\hline Filter to $10 \mathrm{wt} \%$ & sosis & 40.2 & 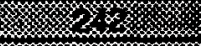 & 0 & 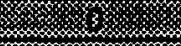 & 0 & stis \\
\hline Stop/Stant & 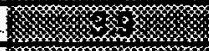 & - & shen & 0 & 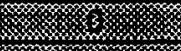 & 0 & mas \\
\hline \multicolumn{8}{|l|}{ Wash Cycle } \\
\hline Wash & sck & 50 & 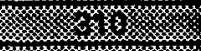 & 2596 & s.t. & 4974 & sy \\
\hline Add $\mathrm{NaOH}$ & 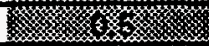 & 8 & st & 498 & 8 & 5687 & 烈 \\
\hline Wash & xy & 70 & $x$ & 7240 & sck & 9045 & scts \\
\hline Add $\mathrm{NaOH}$ & x & 6 & 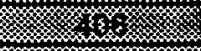 & 647 & 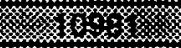 & 9307 & 悴 \\
\hline Wash & x. & 65 & 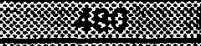 & 7963 & 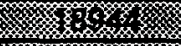 & 11185 & 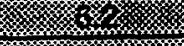 \\
\hline Add NaNO2 & 昰 & 8 & 然 & 999 & 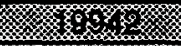 & 11398 & 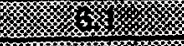 \\
\hline Wash & $x$ & 125 & 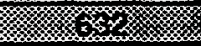 & 19622 & 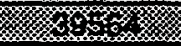 & 10701 & 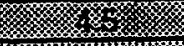 \\
\hline Sample & 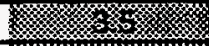 & - & 位 & 4665 & 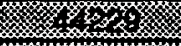 & 6036 & K \\
\hline Transfer Slumy & 玹 & 130 & 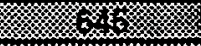 & 827 & 学 & 5209 & 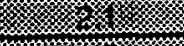 \\
\hline Stop & 卒 & - & 空 & 1515 & 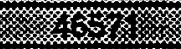 & 3694 & 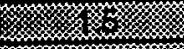 \\
\hline
\end{tabular}

SS = Salt Solution

RWW = Recycled Wash Water

thalicized volume changes indicate removal from Tank 48.

Non-italicized volume changes indicate additions to Tank 48.

The wash cycle volume changes do not cause volume level changes in Tank 48 since the washwater and inhibitor solutions are added and removed simultaneously.

The volume of the contents in Tank 22 is incrementally increased by Late washwater at the rate of 2809 gallons per day. 


\section{APPENDIX A (continued)}

Concentration of TBP Added:

TPB Solublilty in DSS:

TPB Solublilty in Wash Water:

DSS TBP Hydrolysis Rate:

TBP Hydrolysis Rate Constant:
$40 \mathrm{mgh}$

$1.1 \mathrm{mgh}$

$16.0 \mathrm{mg}$.

$0.184 \mathrm{mg} /$ day (it [TBP] > solubility limit)

0.17 /day (if [TBP] < solubility limit)

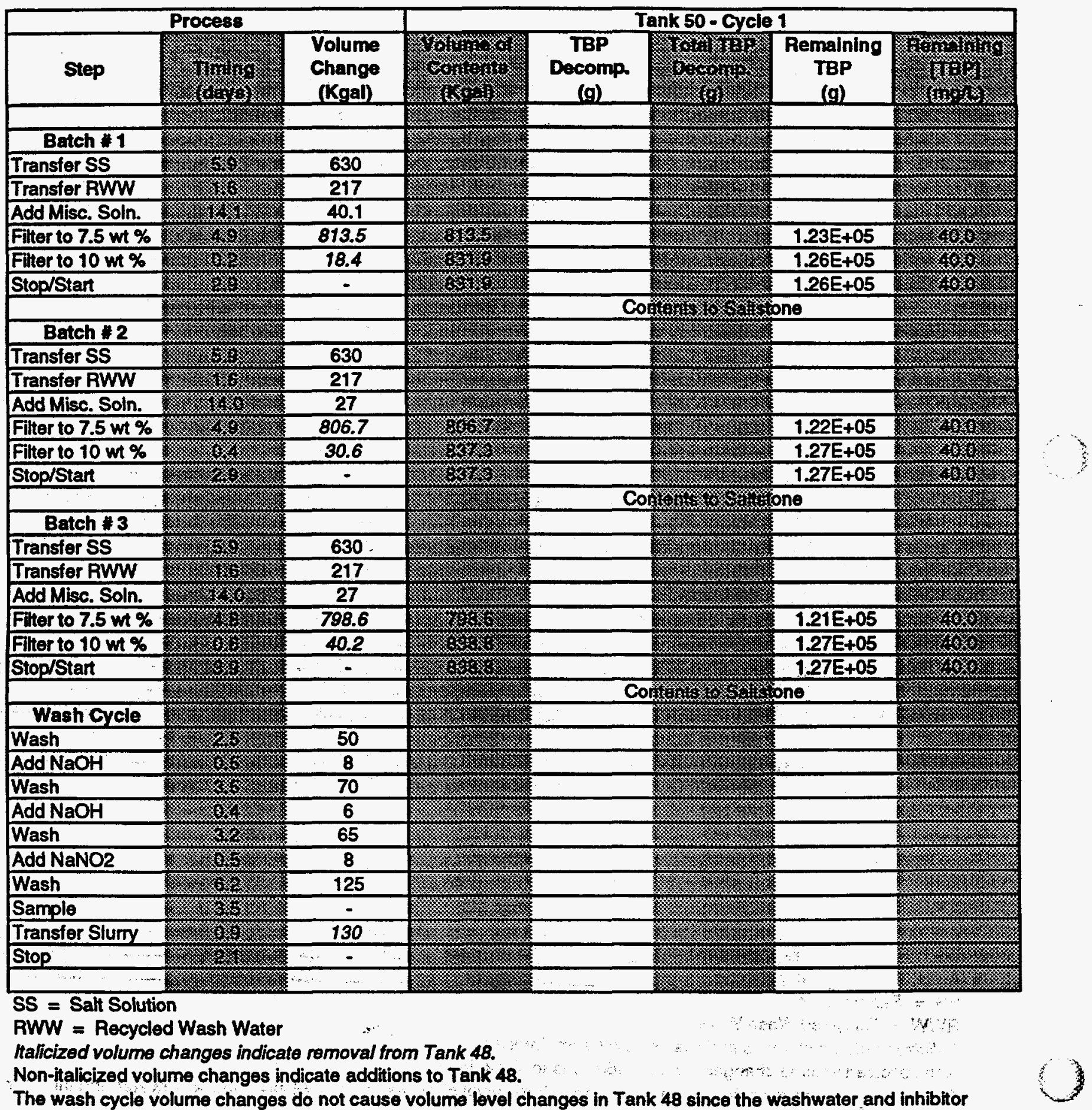

solutions are added and removed simultaneously.

The volume of the contents in Tank 22 is incrementally increased by Late washwater at the rate of 2809 gallons per day. 


\section{APPENDIX A (continued)}

Concentration of TBP Added: TPB Solublithy in DSS:

TPB Solublitty in Wash Water:

DSS TBP Hydrolysis Rate:

TBP Hydrolysls Rate Constant:
$40 \mathrm{mgl}$

$1.1 \mathrm{mgl}$

$16.0 \mathrm{mgl}$

0.184 mgl-day (if [TBP] > solubility limit)

0.17 /day (If [TBP] < solublity limit)

\begin{tabular}{|c|c|c|c|c|c|c|c|}
\hline \multicolumn{3}{|c|}{ Process } & \multicolumn{5}{|c|}{ Tank 48-Cycle 2} \\
\hline Step & 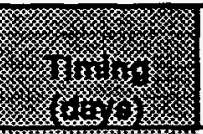 & $\begin{array}{l}\text { Volume } \\
\text { Change } \\
\text { (Kgal) }\end{array}$ & 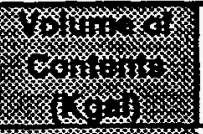 & $\begin{array}{c}\text { TBP } \\
\text { Decomp. } \\
\text { (g) }\end{array}$ & (1) & $\begin{array}{c}\text { Remaining } \\
\text { TBP } \\
\text { (g) } \\
\end{array}$ & 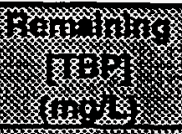 \\
\hline & 侪 & & . & & & & \\
\hline Batch 1 & x & & z & & (x) & & 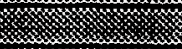 \\
\hline Transfer SS & 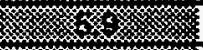 & 630 & 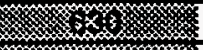 & 0 & 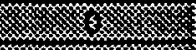 & 0 & - \\
\hline Transfer RWW & 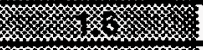 & 217 & 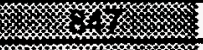 & 0 & m & 743 & 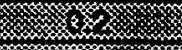 \\
\hline Add Misc. Soln. & $x$ & 40.1 & 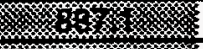 & 671 & $x$ & 73 & 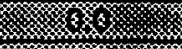 \\
\hline Filter to $7.5 \mathrm{wt} \%$ & m & 813.5 & 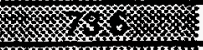 & 70 & 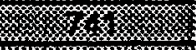 & 0 & 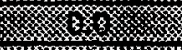 \\
\hline Filter to $10 \mathrm{wt} \%$ & Syzox & 18.4 & . & 0 & 泣 & 0 & s.t. \\
\hline Stop/Start & 好 & - & . & 0 & 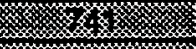 & 0 & x. \\
\hline & & & 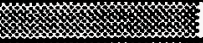 & & (x) & & 组 \\
\hline Batch $\# 2$ & & & . & & 伭 & & 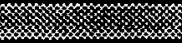 \\
\hline Transfer SS & 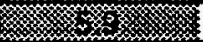 & 630 & 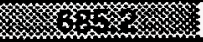 & 0 & 筇 & 0 & 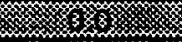 \\
\hline Transfer RWW & 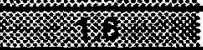 & 217 & w & 0 & . & 4 & 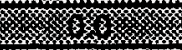 \\
\hline Add Misc. Soln. & 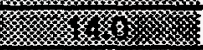 & 27 & Kry & 3 & xys & 0 & 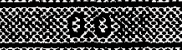 \\
\hline Fitter to $7.5 w t \%$ & 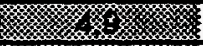 & 806.7 & XYs & 0 & Kas & 0 & X \\
\hline Filter to $10 \mathrm{wt} \%$ & 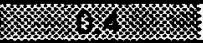 & 30.6 & 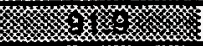 & 0 & 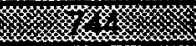 & 0 & 灭 \\
\hline Stop/Start & X & - & 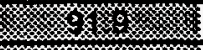 & 0 & 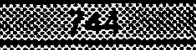 & 0 & 6xick \\
\hline & $x$ & & 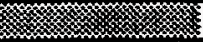 & & 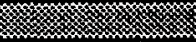 & & 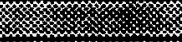 \\
\hline Batch \#3 & & & 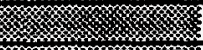 & & $x$ & & \\
\hline Transfer SS & 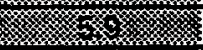 & 630 & $x$ & 0 & . & 0 & 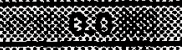 \\
\hline Transfer RWW & t & 217 & 98 & $\overline{0}$ & s & 0 & 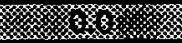 \\
\hline Add Misc. Soln. & \% & 27 & . & $\overline{0}$ & 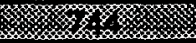 & 0 & zot \\
\hline Filter to $7.5 \mathrm{wt} \%$ & k & 798.6 & 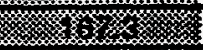 & 0 & 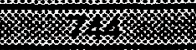 & 0 & x/ \\
\hline Filter to $10 w$ t & (x) & 40.2 & 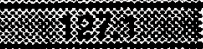 & 0 & 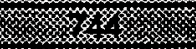 & 0 & 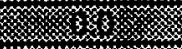 \\
\hline Stop/Start & 帘 & - & 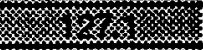 & 0 & 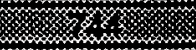 & 0 & $x^{3}$ \\
\hline & & & 弦 & & 文 & & 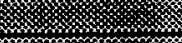 \\
\hline Wash Cycle & 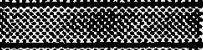 & & 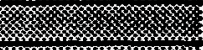 & & 新 & & 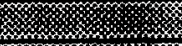 \\
\hline Wash & 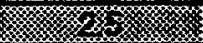 & 50 & xis & $\overline{0}$ & 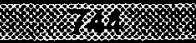 & o & $x$ \\
\hline Add $\mathrm{NaOH}$ & xyct & 8 & 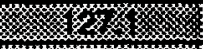 & 0 & 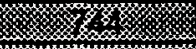 & 0 & 媇 \\
\hline Wash & 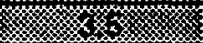 & 70 & 好媇 & $\mathbf{0}$ & 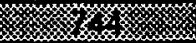 & 0 & 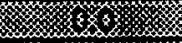 \\
\hline Add $\mathrm{NaOH}$ & 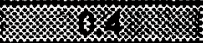 & 6 & 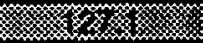 & $\mathbf{0}$ & 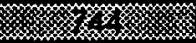 & 0 & 6 \\
\hline Wash & 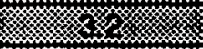 & 65 & k & 0 & w & 0 & 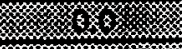 \\
\hline Add $\mathrm{NaNO}_{2}$ & 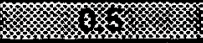 & 8 & 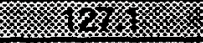 & 0 & 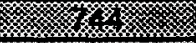 & 0 & 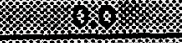 \\
\hline Wash & 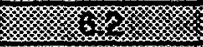 & 125 & 1. & $\overline{0}$ & 努 & $\overline{0}$ & s \\
\hline Sample & 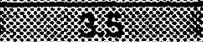 & - & 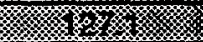 & $\overline{0}$ & Xn & $\overline{0}$ & mon \\
\hline Transfer Slumy & 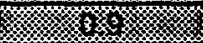 & 130 & ( & $\overline{0}$ & 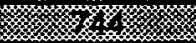 & $\mathbf{0}$ & m \\
\hline Stop & x & - & k & 0 & W & 0 & 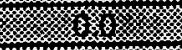 \\
\hline & 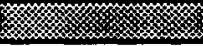 & & 样 & & 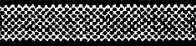 & & 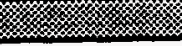 \\
\hline
\end{tabular}

SS = Salt Solution

RWW = Recycled Wash Water

Halicized volume changes indicate removal from Tank 48.

Non-italicized volume changes indicate additions to Tank 48.

The wash cycle volume changes do not cause volume level changes in Tank 48 since the washwater and inhibitor solutions are added and removed simultaneously.

The volume of the contents in Tank 22 is incrementally increased by Late washwater at the rate of 2809 gallons per day. 


\section{APPENDIX A (continued)}

Concentration of TBP Added: TPB Solubility in DSS:

TPB Solublilty In Wach Water:

RWW TBP Hydrolycle Rate:

TBP Hydrolysis Rate Conetant:
$40 \mathrm{mgl}$

$1.1 \mathrm{mgh}$

$16.0 \mathrm{mgh}$

2.679 molloday (if [TBP] > solubllity IImit)

0.17 /day

(If [TBP] < solubllity limit)

\begin{tabular}{|c|c|c|c|c|c|c|c|}
\hline \multicolumn{3}{|c|}{ Proces: } & \multicolumn{5}{|c|}{ Tank 22 - Cycle 2} \\
\hline Step & 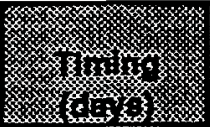 & $\begin{array}{c}\text { Volume } \\
\text { Change } \\
\text { (Kgal) }\end{array}$ & 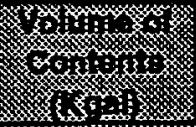 & $\begin{array}{c}\text { TBP } \\
\text { Decomp. } \\
\text { (g) }\end{array}$ & (n) & $\begin{array}{c}\text { Remalning } \\
\text { TBP } \\
\text { (g) }\end{array}$ & (6) \\
\hline & . & & - & & -1 & & \\
\hline Batch 1 & 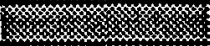 & & . & & & & \\
\hline Transfer SS & 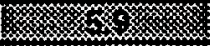 & 630 & 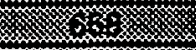 & 1407 & 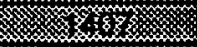 & 2287 & 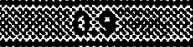 \\
\hline Transfer RWW & 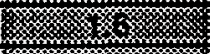 & 217 & x & 1096 & xy & 804 & 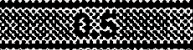 \\
\hline Add Misc. Soln. & 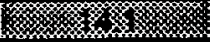 & 40.1 & w & 722 & $4 ;$ & 82 & 筇 \\
\hline Filter to 7.5 wt $\%$ & . & 813.5 & 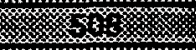 & 45 & 秋 & 37 & . \\
\hline Filter to $10 \mathrm{wt} \%$ & 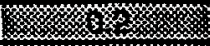 & 18.4 & 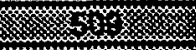 & 1 & cts & 36 & x \\
\hline Stop/Start & 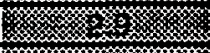 & $=$ & 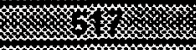 & 13 & 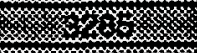 & 22 & . \\
\hline & 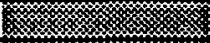 & & x & & 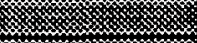 & & 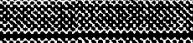 \\
\hline Batch $\# 2$ & $x$ & & 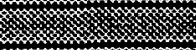 & & 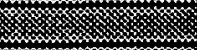 & & $\ldots$ \\
\hline Transfer SS & s. & 630 & 管 & 14 & 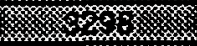 & 9 & 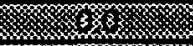 \\
\hline Transfer RWW & x & 217 & -2 & 5 & s. & 2 & 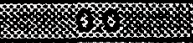 \\
\hline Add Misc. Soln. & 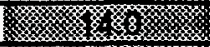 & 27 & . & 2 & 4 & 0 & 的地 \\
\hline Filter to $7.5 w t \%$ & , & 806.7 & 6 & 0 & 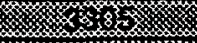 & 0 & $<$ \\
\hline Filter to $10 \mathrm{~m} \%$ & 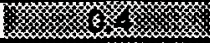 & 30.6 & 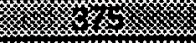 & 0 & 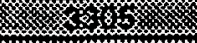 & 0 & (6) \\
\hline Stop/Stant & 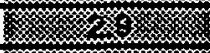 & - & - & 0 & x & 0 & - \\
\hline & & & m & & $x_{x}$ & & 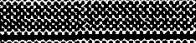 \\
\hline Batch \# 3 & 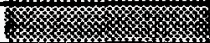 & & & & & & - \\
\hline Transfer SS & 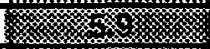 & 630 & 壮 & 0 & 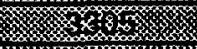 & 0 & 位 \\
\hline Transfer RWW & 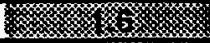 & 217 & 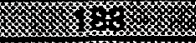 & 0 & - & 0 & 3 \\
\hline Add Misc. Soln. & > & 27 & \% & 0 & 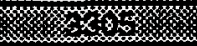 & 0 & 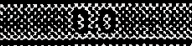 \\
\hline Filter to 7.5 wt \% & 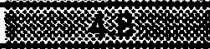 & 798.6 & 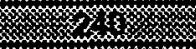 & D & 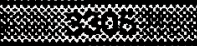 & 0 & 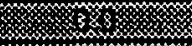 \\
\hline Filter to 10 wt \% & 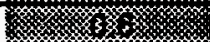 & 40.2 & 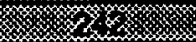 & 0 & 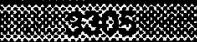 & 0 & 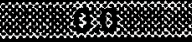 \\
\hline Stop/Start & 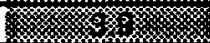 & - & . & 0 & 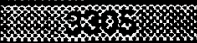 & 0 & t \\
\hline & x & & . & & 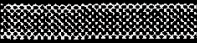 & & x) \\
\hline Wash Cycle & 1 & & & & & & \\
\hline Wash & $x$ & 50 & st & 2596 & wh & 4974 & K) \\
\hline Add $\mathrm{NaOH}$ & 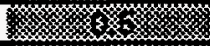 & 8 & w & 498 & 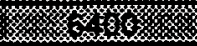 & 5687 & 纹 \\
\hline Wash & 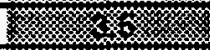 & 70 & sty & 7240 & ry & 9045 & 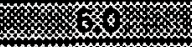 \\
\hline Add $\mathrm{NaOH}$ & 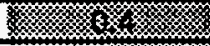 & 6 & w & 647 & 等 & 9307 & 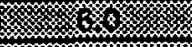 \\
\hline Wash & x & 65 & 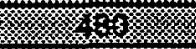 & 7963 & 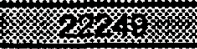 & 11185 & 䇣 \\
\hline Add NaNO2 & 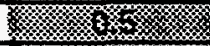 & 8 & 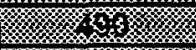 & 999 & $8.258 .58=$ & 11398 & 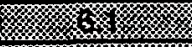 \\
\hline Wash & 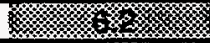 & 125 & $x$ & 19622 & 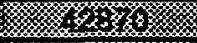 & 10701 & 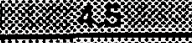 \\
\hline Sample & 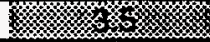 & - & 26 & 4665 & 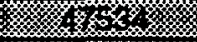 & 6036 & 的 \\
\hline Transfer Slumy & 1 & 130 & 6 & 827 & 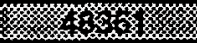 & 5209 & 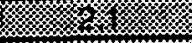 \\
\hline Stop & 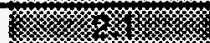 & - & 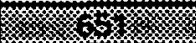 & 1515 & 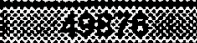 & 3694 & 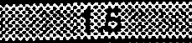 \\
\hline & 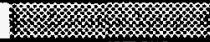 & & 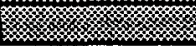 & & 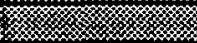 & 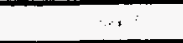 & 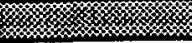 \\
\hline
\end{tabular}

SS = Salt Solution

RWW = Recycled Wash Water

Halicized volume changes indicate removal from Tank 48 .

Non-italicized volume changes indicate additions to Tank 48.

The wash cycle volume changes do not cause volume level changes in Tank 48 since the washwater and inhibitor solutions are added and removed simultaneously.

The volume of the contents in Tank 22 is incrementally increased by Late washwater at the rate of 2809 gallons per day. 


\section{APPENDIX A (continued)}

\section{Concentration of TBP Added: TPB Solubllity in DSS: \\ TPB Solubility in Wash Water: \\ DSS TBP Hydrolycls Rate: \\ TBP Hydrolysls Rate Conetant:}

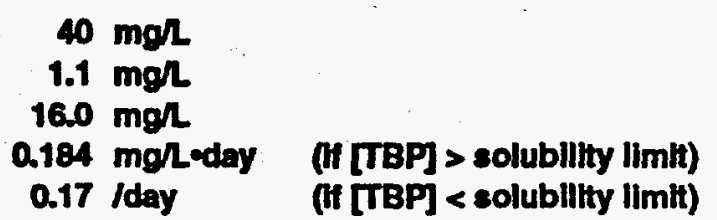

\begin{tabular}{|c|c|c|c|c|c|c|c|}
\hline \multicolumn{3}{|c|}{ Process } & \multicolumn{5}{|c|}{ Tank 50 - Cycle 2} \\
\hline Step & 等 & $\begin{array}{c}\text { Volume } \\
\text { Change } \\
\text { (Kgal) }\end{array}$ & (n) & $\begin{array}{c}\text { TBP } \\
\text { Decomp. } \\
\text { (g) }\end{array}$ & 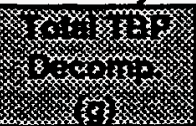 & $\begin{array}{c}\text { Remaining } \\
\text { TBP } \\
\text { (g) }\end{array}$ & 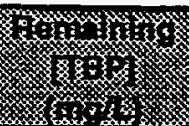 \\
\hline & 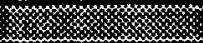 & & -4 & & & & \\
\hline Batch 1 & 暗 & & 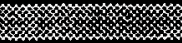 & & 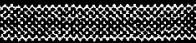 & & \\
\hline Transfer SS & $x$ & 630 & 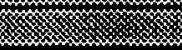 & & 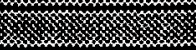 & & $x$ \\
\hline Transfer RWW & 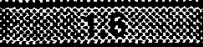 & 217 & 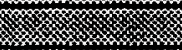 & & & $\cdots$ & xy \\
\hline Add Misc. Soln. & 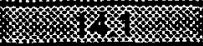 & 40.1 & $x$ & & 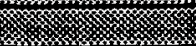 & $\cdot$ & \\
\hline Filter to $7.5 \mathrm{wt} \%$ & xys & 813.5 & 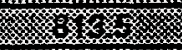 & & $\ldots$ & $1.23 E+05$ & 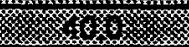 \\
\hline Filter to $10 \mathrm{wt} \%$ & sk & 18.4 & z & & $m$ & $1.26 E+05$ & 枚 \\
\hline Stop/Start & 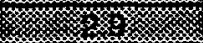 & - & . & & (1) & $1.26 E+05$ & 梦好 \\
\hline & & & 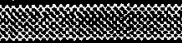 & & $\left(y_{k}\right.$ & ne & \\
\hline Batch 2 & 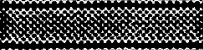 & & 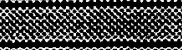 & & m) & & \\
\hline Transfer SS & m & 630 & $m$ & & 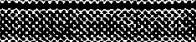 & & \\
\hline Transfer RWW & 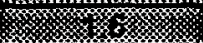 & 217 & 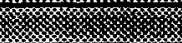 & & & & \\
\hline Add Misc. Soln. & s.s. & 27 & 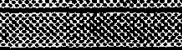 & & & & \\
\hline Filter to 7.5 wt $\%$ & 好 & 806.7 & w & & & $1.22 E+05$ & (6) \\
\hline Filter to $10 w t \%$ & 原媇 & 30.6 & z & & 伭 & $1.27 E+05$ & 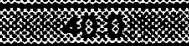 \\
\hline Stop/Start & 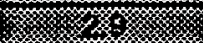 & $=$ & 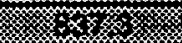 & & 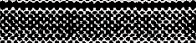 & $1.27 E+05$ & 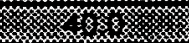 \\
\hline & 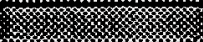 & & 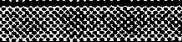 & & mox & ne & \\
\hline Batch 3 & $x$ & & $x$ & & 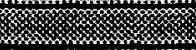 & & \\
\hline Transfer SS & xy & 630 & 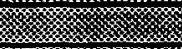 & & & & 然 \\
\hline Transfer RWW & (3) & 217 & 1x & & $3 x$ & & \\
\hline Add Misc. Soln. & 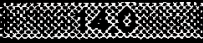 & 27 & $\ldots$ & & 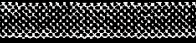 & & 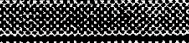 \\
\hline Fither to 7.5 wt $\%$ & 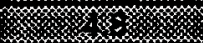 & 798.6 & 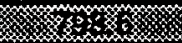 & & & $1.21 E+05$ & 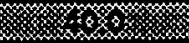 \\
\hline Fither to $10 w$ \% & 䇣 & 40.2 & 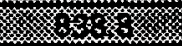 & & 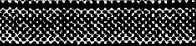 & $1.27 E+05$ & 警 \\
\hline Stop/Stant & $2 \times$ & $=$ & 的 & & 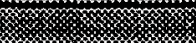 & $1.27 E+05$ & 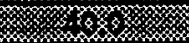 \\
\hline & 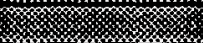 & & 耍 & & 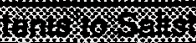 & ne & \\
\hline Wash Cycle & 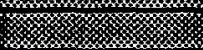 & & X & & 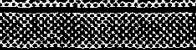 & & \\
\hline Wash & 2 & 50 & 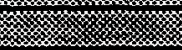 & & & & \\
\hline Add $\mathrm{NaOH}$ & 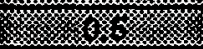 & 8 & kx & & 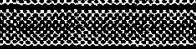 & & \\
\hline Wash & 统 & 70 & 妆 & & & & \\
\hline Add $\mathrm{NaOH}$ & 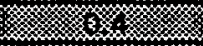 & 6 & m. & & 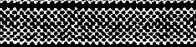 & & \\
\hline Wash & 4 & 65 & & & & & \\
\hline Add NaNO2 & 然 & 8 & & & & & \\
\hline Wash & 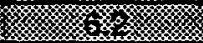 & 125 & & & & & 2 \\
\hline Sample & 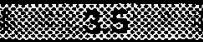 & - & 柇 & & & & 3. \\
\hline Transfer Slumy & X & 130 & 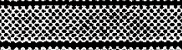 & & (ry & & 柿 \\
\hline Stop & 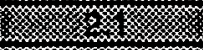 & - & 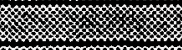 & & 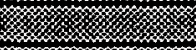 & & 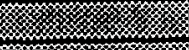 \\
\hline & 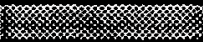 & & 媇 & & 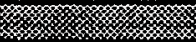 & & 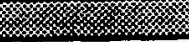 \\
\hline
\end{tabular}

SS = Salt Solution

RWW = Recycled Wash Water

ftalicized volume changes indicate removal from Tank 48.

Non-ftalicized volume changes indicate additions to Tank 48.

The wash cycle volume changes do not cause volume level changes in Tank $\mathbf{4 8}$ since the washwater and inhibitor solutions are added and removed simultaneously.

The volume of the contents in Tank 22 is incrementally increased by Late washwater at the rate of 2809 gallons per day. 


\section{APPENDIX A (continued)}

\begin{abstract}
Concentration of TBP Added: TPB Solublitty in DSS:

TPB Solublitty In Wash Water:

DSS TBP Hydrolysis Rate:

TBP Hydrohysls Rate Constant:
\end{abstract}

\author{
$40 \mathrm{mgh}$ \\ $1.1 \mathrm{mgh}$ \\ $16.0 \mathrm{mgh}$ \\ 0.184 mglleday \\ 0.17 /day
}

(f [TBP] > solublity limit)

(H [TBP] < solubllity IImit)

\begin{tabular}{|c|c|c|c|c|c|c|c|}
\hline \multicolumn{3}{|c|}{ Process } & \multicolumn{5}{|c|}{ Tank 48 - Cycle 3} \\
\hline Step & 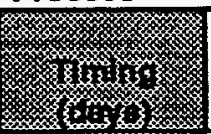 & $\begin{array}{c}\text { Volume } \\
\text { Change } \\
\text { (Kgal) }\end{array}$ & 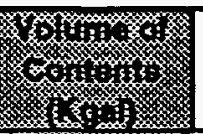 & $\begin{array}{c}\text { TBP } \\
\text { Docomp. } \\
\text { (g) }\end{array}$ & Y.t. & $\begin{array}{c}\text { Remaining } \\
\text { TBP } \\
\text { (g) }\end{array}$ & (1) \\
\hline & 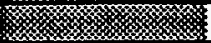 & & & & & & 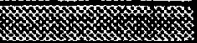 \\
\hline Batch $* 1$ & 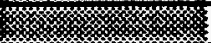 & & $\ldots$ & & $\ldots$ & & \\
\hline Transfer SS & 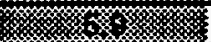 & 630 & 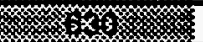 & 0 & m & $\overline{0}$ & 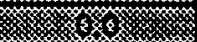 \\
\hline Transfer RWW & 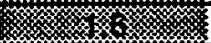 & 217 & 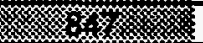 & $\overline{0}$ & (2) & 743 & 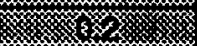 \\
\hline Add Misc. Soln. & 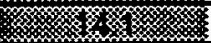 & 40.1 & 等 & 671 & w & 73 & 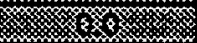 \\
\hline Filter to $7.5 w t \%$ & 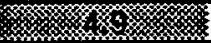 & 813.5 & 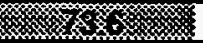 & 70 & 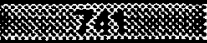 & 0 & 等 \\
\hline Filter to $10 \mathrm{wt} \%$ & ris & 18.4 & (3) & 0 & $\ldots$ & $\overline{0}$ & (6) \\
\hline Stop/Start & $x_{x}$ & - & c & $\overline{0}$ & 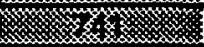 & $\overline{0}$ & 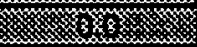 \\
\hline & 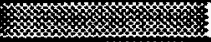 & & 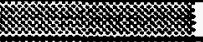 & & $\ldots$ & & $\ldots$ \\
\hline Batch $* 2$ & 伭 & & 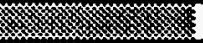 & & 柇 & & $\ldots$ \\
\hline Transfer SS & 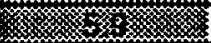 & 630 & (z) & $\overline{0}$ & 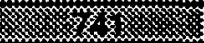 & $\overline{0}$ & 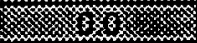 \\
\hline Transfer RWW & 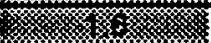 & 217 & $\ldots$ & $\overline{0}$ & 算 & 4 & m \\
\hline Add Misc. Soln. & 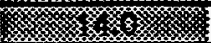 & 27 & s.s.s. & 3 & (3) & $\overline{0}$ & 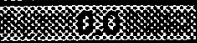 \\
\hline Filter to 7.5 wt $\%$ & X/. & 806.7 & $\ldots$ & 0 & \% & $\overline{0}$ & 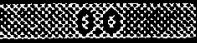 \\
\hline Filter to $10 w t \%$ & ( & 30.6 & \% & 0 & 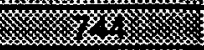 & $\overline{0}$ & z \\
\hline Stop/Stant & 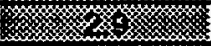 & - & 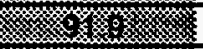 & $\overline{0}$ & 柇 & $\overline{0}$ & 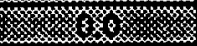 \\
\hline & $m$ & & 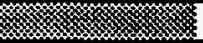 & & x & & \\
\hline Batch \# 3 & $x$ & & . & & & & 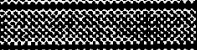 \\
\hline Transfer SS & m & 630 & scksck & 0 & (8) & 0 & 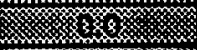 \\
\hline Transier RWW & 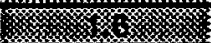 & 217 & (1) & $\overline{0}$ & (6) & $\overline{0}$ & 3 \\
\hline Add Misc. Soln. & X & 27 & (1) & $\overline{0}$ & \% & $\overline{0}$ & - \\
\hline Filter to $7.5 \mathrm{wt} \%$ & - & 798.6 & 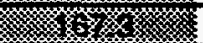 & $\overline{0}$ & \% & $\overline{0}$ & 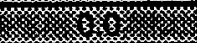 \\
\hline Filter to $10 \mathrm{wt} \%$ & w & 40.2 & 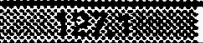 & 0 & 多 & 0 & 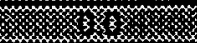 \\
\hline Stop/Start & 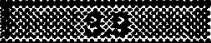 & - & 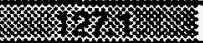 & 0 & 簤 & 0 & 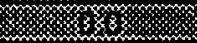 \\
\hline & 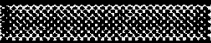 & & $\ldots$ & & 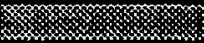 & & 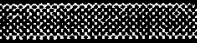 \\
\hline Wash Cycle & 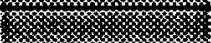 & & ry & & 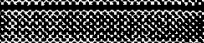 & & 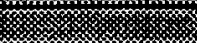 \\
\hline Wash & 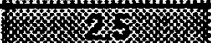 & 50 & 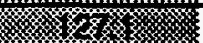 & $\overline{0}$ & 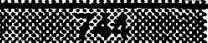 & $\overline{0}$ & 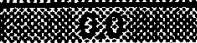 \\
\hline Add $\mathrm{NaOH}$ & 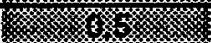 & 8 & 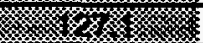 & $\overline{0}$ & 16 & $\overline{0}$ & 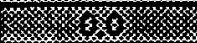 \\
\hline Wash & 1) & 70 & 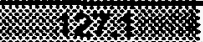 & $\overline{0}$ & 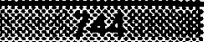 & 0 & 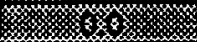 \\
\hline Add $\mathrm{NaOH}$ & 帘 & 6 & ( & 0 & $1 \times$ & 0 & 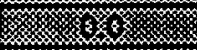 \\
\hline Wash & $\gamma_{x}$ & 65 & 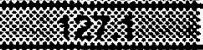 & 0 & k & 0 & 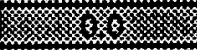 \\
\hline Add NaNO2 & 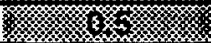 & 8 & 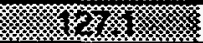 & $\mathbf{0}$ & w & 0 & ry \\
\hline Wash & 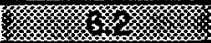 & 125 & (6) & $\mathbf{0}$ & 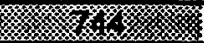 & 0 & 展, \\
\hline Sample & 㒀 & - & (6) & $\overline{0}$ & ry & 0 & 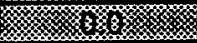 \\
\hline Transfier Slumy & (4) & 130 & 3 & 0 & 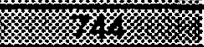 & 0 & 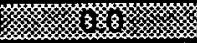 \\
\hline Stop & 级 & - & 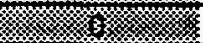 & 0 & 秋 & $\overline{0}$ & 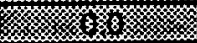 \\
\hline & ' & & 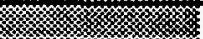 & & 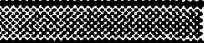 & & 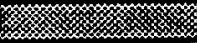 \\
\hline
\end{tabular}

SS $=$ Salt Solution

RWW = Recycled Wash Water

Halicized volume changes indicate removal from Tank 48.

Non-italicized volume changes indicate additions to Tank 48.

The wash cycle volume changes do not cause volume level changes in Tank 48 since the washwater and inhibitor solutions are added and removed simultaneously.

The volume of the contents in Tank 22 is incrementally increased by Late washwater at the rate of 2809 gallons per day. 


\section{APPENDIX A (continued)}

Concentration of TBP Added: TPB Solublilty in DSS:

TPB Solubility in Wash Water:

RWW TBP Hydrolysls Rate:

TBP Hydrolysle Rate Constant:

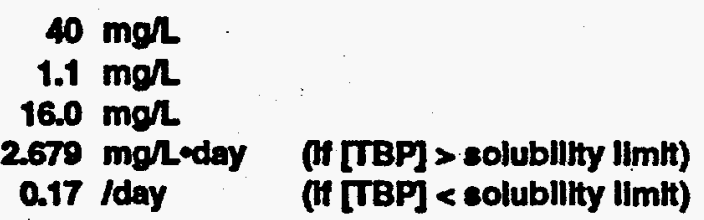

\begin{tabular}{|c|c|c|c|c|c|c|c|}
\hline \multicolumn{3}{|c|}{ Process } & \multicolumn{5}{|c|}{ Tank 22 - Cycle 3} \\
\hline Step & 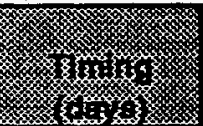 & $\begin{array}{c}\text { Volume } \\
\text { Change } \\
\text { (Kgal) }\end{array}$ & (2) & $\begin{array}{c}\text { TBP } \\
\text { Decomp. } \\
\text { (g) }\end{array}$ & ( & $\begin{array}{c}\text { Remaining } \\
\text { TBP } \\
\text { (g) }\end{array}$ & 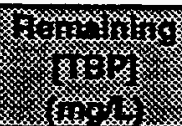 \\
\hline & 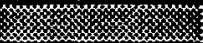 & & 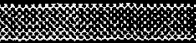 & & 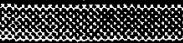 & & . \\
\hline Batch \#1 & & & x & & 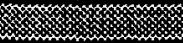 & & 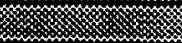 \\
\hline Transfer SS & 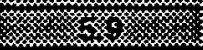 & 630 & 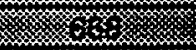 & 1407 & . & 2287 & - \\
\hline Transfer RWW & 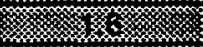 & 217 & $x$ & 1096 & 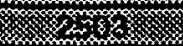 & 804 & 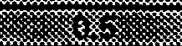 \\
\hline Add Misc. Soln. & 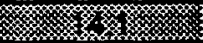 & 40.1 & 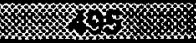 & 722 & 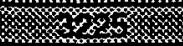 & 82 & 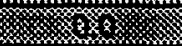 \\
\hline Filter to 7.5 wt $\%$ & 玹 & 813.5 & 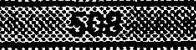 & 45 & (3) & 37 & 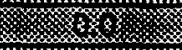 \\
\hline Fitter to 10 wt \% & s. & 18.4 & 34 & 1 & $x$ & 36 & 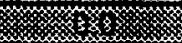 \\
\hline Stop/Start & s. & - & ty & 13 & 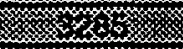 & 22 & 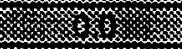 \\
\hline & 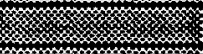 & & xy & & 媇 & & my \\
\hline Batch 2 & 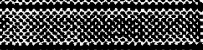 & & & & 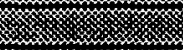 & & $x$ \\
\hline Transfer SS & s & 630 & 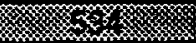 & 14 & $\alpha$ & 9 & 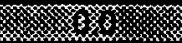 \\
\hline Transfer RWW & 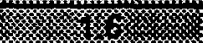 & 217 & 好 & 5 & $x y$ & 2 & 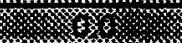 \\
\hline Add Misc. Soln. & 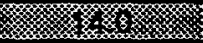 & 27 & tot & 2 & 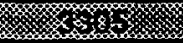 & 0 & 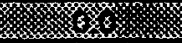 \\
\hline Filter to $7.5 \mathrm{wt} \%$ & 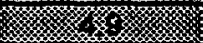 & 806.7 & 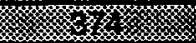 & 0 & 4 & 0 & 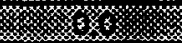 \\
\hline Filter to $10 \mathrm{wt} \%$ & 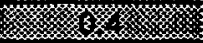 & 30.6 & -2 & 0 & 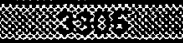 & 0 & 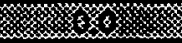 \\
\hline Stop/Start & 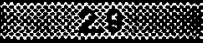 & - & 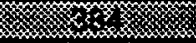 & 0 & 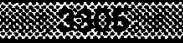 & 0 & 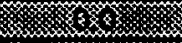 \\
\hline & & & 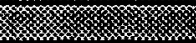 & & 新 & & m, my \\
\hline Batch \# 3 & m & & & & 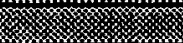 & & 氺 \\
\hline Transfer SS & 1. & 630 & 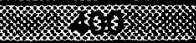 & 0 & 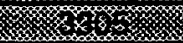 & 0 & stis \\
\hline Transier RWW & xy & 217 & $4 x$ & 0 & 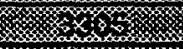 & 0 & 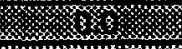 \\
\hline Add Misc. Soln. & 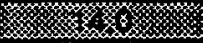 & 27 & 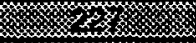 & 0 & 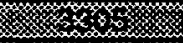 & 0 & 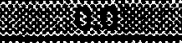 \\
\hline Filter to 7.5 wt \% & 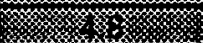 & 798.6 & 等 & $\overline{0}$ & s. & $\overline{0}$ & m \\
\hline Filter to $10 w t \%$ & 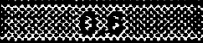 & 40.2 & 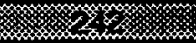 & 0 & 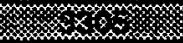 & 0 & 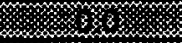 \\
\hline Stop/Start & 好好 & $=$ & 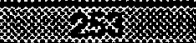 & 0 & 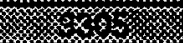 & 0 & m \\
\hline & & & 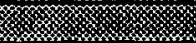 & & & & \\
\hline Wash Cycle & m. & & 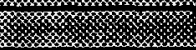 & & xy & $\ldots$ & (x) \\
\hline Wash & $\langle x$ & 50 & \% & 2596 & . & 4974 & 级级 \\
\hline Add $\mathrm{NaOH}$ & 繁 & 8 & 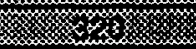 & 498 & 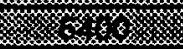 & 5687 & m \\
\hline Wash & (x) & 70 & 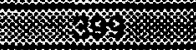 & 7240 & 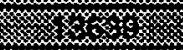 & 9045 & m \\
\hline Add $\mathrm{NaOH}$ & s. & 6 & 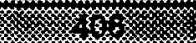 & 647 & 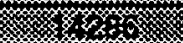 & 9307 & 筑 \\
\hline Wash & 好 & 65 & 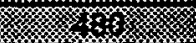 & 7963 & st & 11185 & 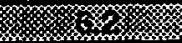 \\
\hline Add NaNO2 & ras & 8 & 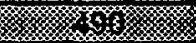 & 999 & 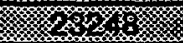 & 11398 & 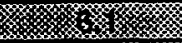 \\
\hline Wash & 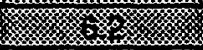 & 125 & 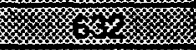 & 19622 & $x$ & 10701 & 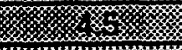 \\
\hline Sample & Kxys & - & x & 4665 & 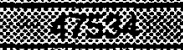 & 6036 & 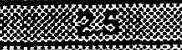 \\
\hline Transfer Slumy & 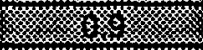 & 130 & x & 827 & 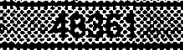 & 5209 & 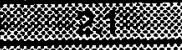 \\
\hline Stop & 校 & - & 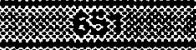 & 1515 & 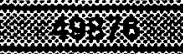 & 3694 & 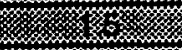 \\
\hline & - & & 留 & & 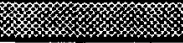 & & 位 \\
\hline
\end{tabular}

SS = Salt Solution

RWW = Recycled Wash Water

Italicized volume changes indicate removal from Tank 48.

Non-italicized volume changes indicate additions to Tank 48 .

The wash cycle volume changes do not cause volume level changes in Tank 48 since the washwater and inhibitor solutions are added and removed simultaneously.

The volume of the contents in Tank 22 is incrementally increased by Late washwater at the rate of 2809 gallons per day. 
APPENDIX A (continued)

\section{Concentration of TBP Added: TPB Solublilty in DSS: \\ TPB Solublilty in Wash Water: DSS TBP Hydrolysle Rate: TBP Hydrolysis Rate Constant:}

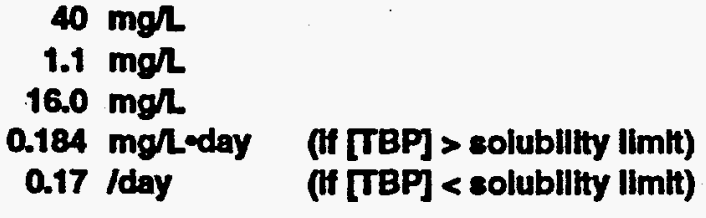

\begin{tabular}{|c|c|c|c|c|c|c|c|}
\hline \multicolumn{3}{|c|}{ Process } & \multicolumn{5}{|c|}{ Tank 50 - Cycle 3} \\
\hline Step & 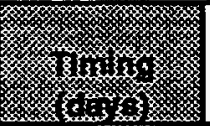 & $\begin{array}{l}\text { Volume } \\
\text { Change } \\
\text { (Kgal) }\end{array}$ & (1) & $\begin{array}{c}\text { TBP } \\
\text { Decomp. } \\
\text { (g) }\end{array}$ & 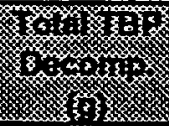 & $\begin{array}{c}\text { Remaining } \\
\text { TBP } \\
\text { (g) }\end{array}$ & (T) \\
\hline & 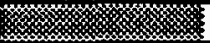 & & - & & $\ldots$ & & whe \\
\hline Batch 1 & 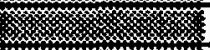 & & 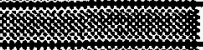 & & 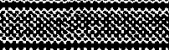 & & \\
\hline Transfer SS & 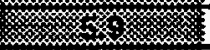 & 630 & 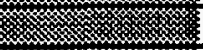 & & 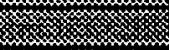 & & 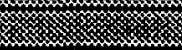 \\
\hline Transfer BWW & 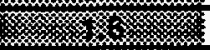 & 217 & cy & & 空 & & \\
\hline Add Misc. Soln. & 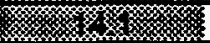 & 40.1 & 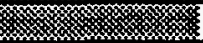 & & 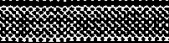 & & \\
\hline Filter to 7.5 wt \% & 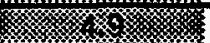 & 813.5 & 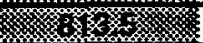 & & $x$ & $1.23 E+05$ & (6) \\
\hline Filter to 10 wt \% & - & 18.4 & . & & & $1.26 E+05$ & 校 \\
\hline Stop/Start & 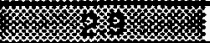 & - & \% & & 校 & $1.26 E+05$ & 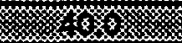 \\
\hline & & & 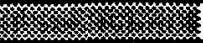 & & mo & ne & \\
\hline Batch & 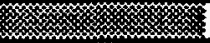 & & 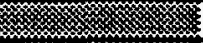 & & 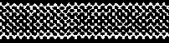 & & 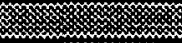 \\
\hline Transfer SS & 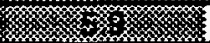 & 630 & 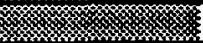 & & 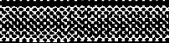 & & 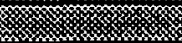 \\
\hline Transfer RWW & 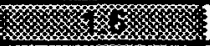 & 217 & 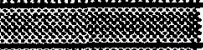 & & 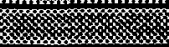 & & \\
\hline Add Misc. Soln. & 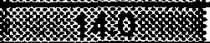 & 27 & . & & & & \\
\hline Filter to 7.5 wt \% & 6 & 806.7 & tist & & & $1.22 E+05$ & 2 \\
\hline Fitter to $10 \mathrm{wt} \%$ & 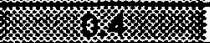 & 30.6 & ( & & . & $1.27 E+05$ & w \\
\hline Stop/Start & 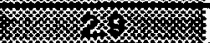 & - & 好留 & & 媇 & $1.27 E+05$ & 柇 \\
\hline & 㡭 & & 留 & & 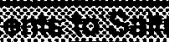 & ne & \\
\hline Batch $\because 3$ & 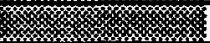 & & 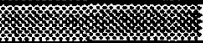 & & 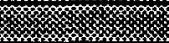 & & 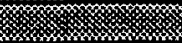 \\
\hline Transfer SS & x. & 630 & $x$ & & & & \\
\hline Transfer RWW & 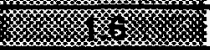 & 217 & 奴 & & . & & . \\
\hline Add Misc. Soln. & $\psi_{k}^{\prime}$ & 27 & W. & & 1/. & & (x) \\
\hline Filter to 7.5 wt \% & 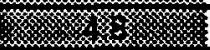 & 798.6 & 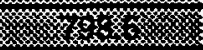 & & 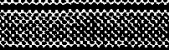 & $1.21 E+05$ & 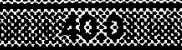 \\
\hline Filter to $10 \mathrm{wt} \%$ & W & 40.2 & 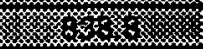 & & $\bar{x}_{x}$ & $1.27 E+05$ & 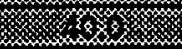 \\
\hline Stop/Stant & 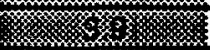 & - & 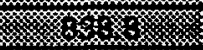 & & 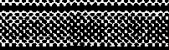 & $1.27 E+05$ & 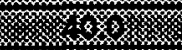 \\
\hline & 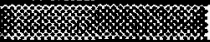 & & 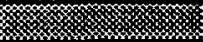 & & - & ne & \\
\hline Wash Cycle & (1) & & \% & & 前济 & & \\
\hline Wash & 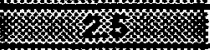 & 50 & 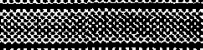 & & 纹玹 & & \\
\hline Add $\mathrm{NaOH}$ & 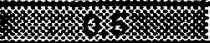 & 8 & 柇 & & 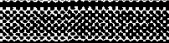 & & 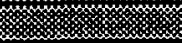 \\
\hline Wash & 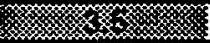 & 70 & 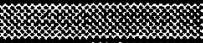 & & 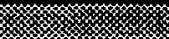 & & 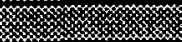 \\
\hline Add $\mathrm{NaOH}$ & 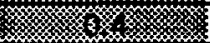 & 6 & 鬲 & & & & 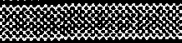 \\
\hline Wash & r. & 65 & 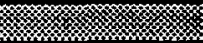 & & m & & 穸 \\
\hline Add NaNO2 & 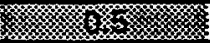 & 8 & 㐬 & & & & $x$ \\
\hline Wash & 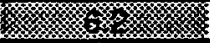 & 125 & 多, & & 器 & & 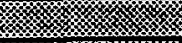 \\
\hline Sample & 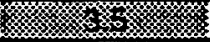 & - & 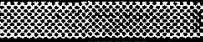 & & xin & & 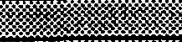 \\
\hline Transfer Slumy & 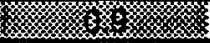 & 130 & & & 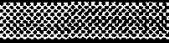 & & (1) \\
\hline Stop & 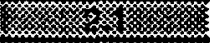 & - & 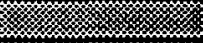 & & 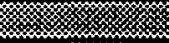 & & 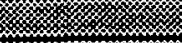 \\
\hline the & 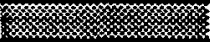 & & 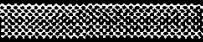 & & 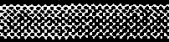 & $\ldots$ & 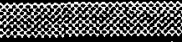 \\
\hline
\end{tabular}

SS = Salt Solution

RWW = Recycled Wash Water

Halicized volume changes indicate removal from Tank 48.

Non-italicized volume changes indicate additions to Tank 48.

The wash cycle volume changes do not cause volume level changes in Tank 48 since the washwater and inhibitor solutions are added and removed simultaneously.

The volume of the contents in Tank 22 is incrementally increased by Late washwater at the rate of 2809 gallons per day. 


\section{APPENDIX B}

The material balance for the addition of $100 \mathrm{mg} / \mathrm{LBP}$ in Tanks 48, 50, and 22 is shown below.

Concentration of TBP Added: TPB Solublity in DSS:

TPB Solublitty In Wash Water:

DSS TBP Hydrolysis Rate:

TBP Hydrolysis Rate Constant:

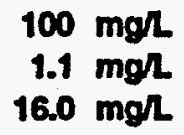

$100 \mathrm{mgh}$

$1.1 \mathrm{mgh}$.

$16.0 \mathrm{mgh}$

$0.184 \mathrm{mgl}$-day (if [TBP] > solubility limit)

0.17 /day (if [TBP] < colubility limit)

\begin{tabular}{|c|c|c|c|c|c|c|c|}
\hline \multicolumn{3}{|c|}{ Process } & \multicolumn{5}{|c|}{ Tank 48 - Cycle 1} \\
\hline Step & 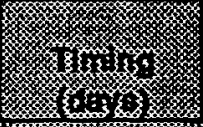 & $\begin{array}{c}\text { Volume } \\
\text { Change } \\
\text { (Kgal) }\end{array}$ & s.t. & $\begin{array}{c}\text { TBP } \\
\text { Decomp. } \\
\text { (g) }\end{array}$ & $x_{13}^{3}$ & $\begin{array}{c}\text { Remaining } \\
\text { TBP } \\
\text { (g) } \\
\end{array}$ & $\frac{1}{20}$ \\
\hline & & & 西 & & & & 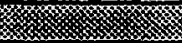 \\
\hline Batch 1 & 1. & & & & 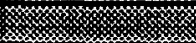 & & 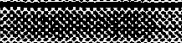 \\
\hline Transfer SS & 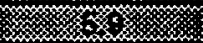 & 630 & 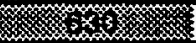 & 0 & 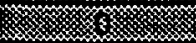 & $\overline{0}$ & 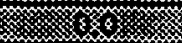 \\
\hline Transfer RWW & 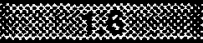 & 217 & 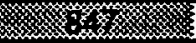 & 0 & $\ldots$ & 0 & 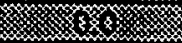 \\
\hline Add Misc. Soln. & 筮 & 40.1 & 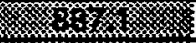 & 0 & 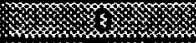 & 0 & 烈 \\
\hline Filter to 7.5 w \% & 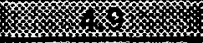 & 813.5 & $x$ & 0 & 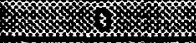 & 0 & 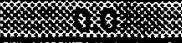 \\
\hline Filter to $10 \mathrm{wt} \%$ & s. & 18.4 & s. & 0 & 3 & 0 & 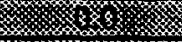 \\
\hline Stop/Stant & sctzons & - & 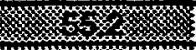 & 0 & 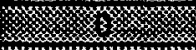 & 0 & s.ts \\
\hline & 给 & & _ & & 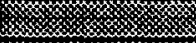 & & 柆 \\
\hline Batch $\because 2$ & 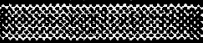 & & $\ldots$ & & 筑 & & 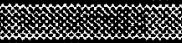 \\
\hline Transfer SS & ry & 630 & 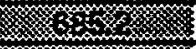 & 0 & 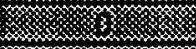 & 0 & (x) \\
\hline Transfer RWW & 偠, & 217 & xy & 0 & s. & 0 & 缕 \\
\hline Add Misc. Soln. & 6 & 27 & 4.45 & 0 & 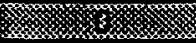 & 0 & s \\
\hline Filter to $7.5 \mathrm{wt} \%$ & 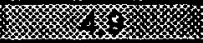 & 806.7 & . & 0 & s. & 0 & 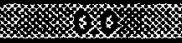 \\
\hline Filter to $10 \mathrm{wt} \%$ & 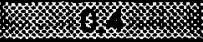 & 30.6 & 1.6. & 0 & 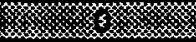 & 0 & 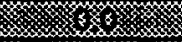 \\
\hline Stop/Start & 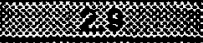 & - & 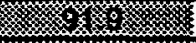 & 0 & 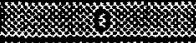 & 0 & (2) \\
\hline & 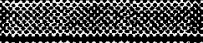 & & - & & 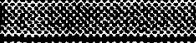 & & 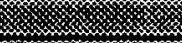 \\
\hline Batch 13 & 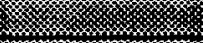 & & (x) & & - & & 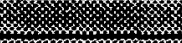 \\
\hline Transfer SS & 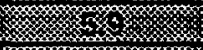 & 630 & 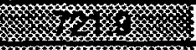 & 0 & 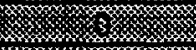 & 0 & 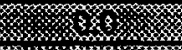 \\
\hline Transfer RWW & 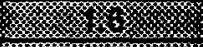 & 217 & 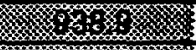 & 0 & 3 & 0 & 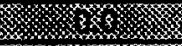 \\
\hline Add Misc. Soln. & 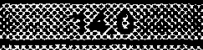 & 27 & 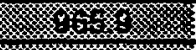 & 0 & 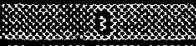 & 0 & 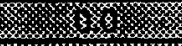 \\
\hline Filter to 7.5 wt \% & X & 798.6 & 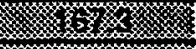 & 0 & 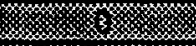 & 0 & 4 \\
\hline Filter to 10 wt \% & 3 & 40.2 & . & 0 & ( & 0 & 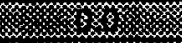 \\
\hline Stop/Start & 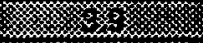 & - & 济 & 0 & 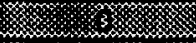 & 0 & 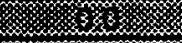 \\
\hline & & & . & & 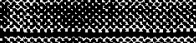 & & (x) \\
\hline Wash Cycle & 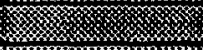 & & . & & 系必 & & x. \\
\hline Wash & (2) & 50 & F & 0 & 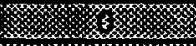 & 0 & 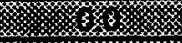 \\
\hline Add $\mathrm{NaOH}$ & 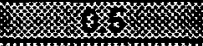 & 8 & 新新 & 0 & 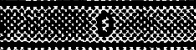 & 0 & 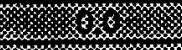 \\
\hline Wash & 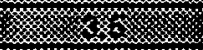 & 70 & 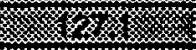 & 0 & (x) & 0 & 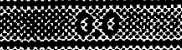 \\
\hline Add $\mathrm{NaOH}$ & 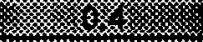 & 6 & 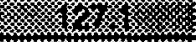 & 0 & 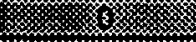 & 0 & 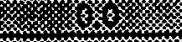 \\
\hline Wash & $x$ & 65 & 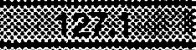 & 0 & 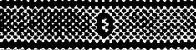 & 0 & 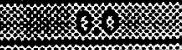 \\
\hline Add $\mathrm{NaNO}_{2}$ & 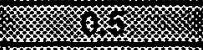 & 8 & 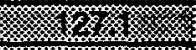 & 0 & 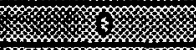 & 0 & 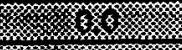 \\
\hline Wash & 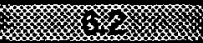 & 125 & (4) & 0 & (1) & 0 & 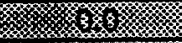 \\
\hline Sample & 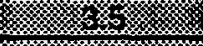 & $=$ & 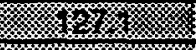 & 0 & ( & 0 & 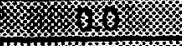 \\
\hline Transfer Slumy & - & 130 & 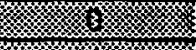 & 0 & $x_{x}$ & 0 & 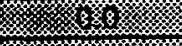 \\
\hline Stop & 2 & $=$ & $3=x$ & 0 & (x) & 0 & 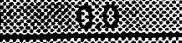 \\
\hline & ; & & 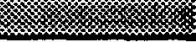 & & , & & 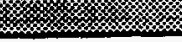 \\
\hline
\end{tabular}

SS = Salt Solution

RWW = Recycled Wash Water

Malicized volume changes indicate removal from Tank 48.

Non-italicized volume changes indicate additions to Tank 48.

The wash cycle volume changes do not cause volume level changes in Tank 48 since the washwater and inhibitor solutions are added and removed simultaneously.

The volume of the contents in Tank 22 is incrementally increased by Late washwater at the rate of $2 \overline{809}$ gallons per day. 
APPENDIX B (continued)

Concentration of TBP Added:
TPB Solubllity in DSS:
TPB Solubllity in Wash Water:
FWW TBP Hydrolysis Rate:
TBP Hydrolysls Rate Constant:

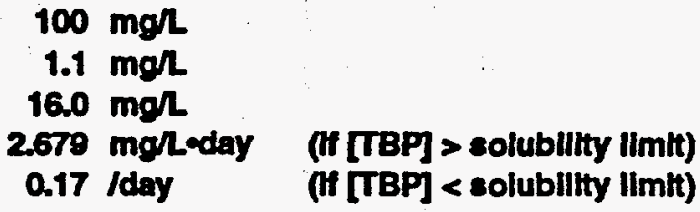

\begin{tabular}{|c|c|c|c|c|c|c|c|}
\hline \multicolumn{3}{|c|}{ Process } & \multicolumn{4}{|c|}{ Tank 22-Cycle 1} & \\
\hline Step & 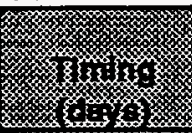 & $\begin{array}{l}\text { Volume } \\
\text { Change } \\
\text { (Kgal) }\end{array}$ & 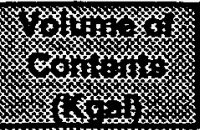 & $\begin{array}{c}\text { TBP } \\
\text { Decomp. } \\
\text { (g) }\end{array}$ & 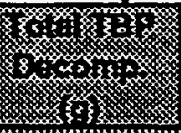 & $\begin{array}{c}\text { Remalning } \\
\text { TBP } \\
\text { (q) }\end{array}$ & mos \\
\hline & & & & & & & \\
\hline Batch 1 & & & & & & & 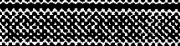 \\
\hline Transfer SS & $\ldots$ & 630 & 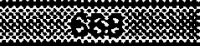 & 0 & cy & 0 & m. \\
\hline Transfer RWW & 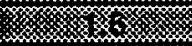 & 217 & w. & 0 & 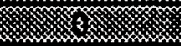 & 0 & 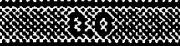 \\
\hline Add Misc. Soln. & 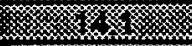 & 40.1 & 列 & 0 & 纹 & 0 & 站 \\
\hline Filter to 7.5 wt $\%$ & s & 813.5 & xys & 0 & $\leqslant$ & 0 & 偠 \\
\hline Filter to 10 wt \% & Ks & 18.4 & w & 0 & 3 & 0 & - \\
\hline Stop/Stant & 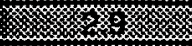 & - & 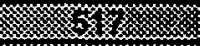 & 0 & m & 0 & W \\
\hline & & & 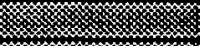 & & & & 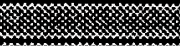 \\
\hline Batch : 2 & 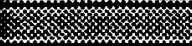 & & my & & 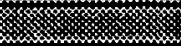 & & 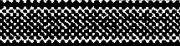 \\
\hline Transfer SS & s. & 630 & s & 0 & 怪 & 0 & 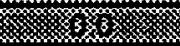 \\
\hline Transter RWW & zons & 217 & x & 0 & Schos & 0 & 答 \\
\hline Add Misc. Soln. & K & 27 & 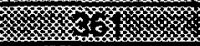 & 0 & $x$ & 0 & m \\
\hline Filter to $7.5 \mathrm{wt} \%$ & 絁 & 806.7 & 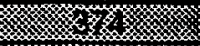 & 0 & s & 0 & . \\
\hline Filter to $10 w t \%$ & s. & 30.6 & th & 0 & 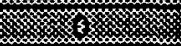 & 0 & . \\
\hline Stop/Start & 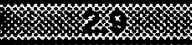 & - & 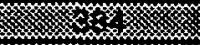 & 0 & 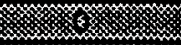 & 0 & 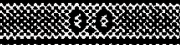 \\
\hline & $x$ & & 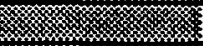 & & x & & \\
\hline Batch 3 & & & $x$ pox & & 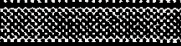 & & $x$ \\
\hline Transter SS & 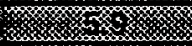 & 630 & 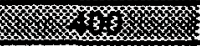 & 0 & sin & 0 & 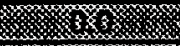 \\
\hline Transfer RWW & 级 & 217 & xy & 0 & 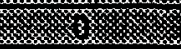 & 0 & ms \\
\hline Add Misc. Soln. & K & 27 & cy & 0 & 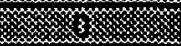 & 0 & 裂 \\
\hline Fitter to 7.5 wt \% & x & 798.6 & x & 0 & 3 & 0 & 柆 \\
\hline Filter to $10 \mathrm{wt} \%$ & 积 & 40.2 & $x$ & 0 & 3 & 0 & m \\
\hline Stop/Stan & 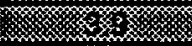 & - & 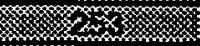 & 0 & 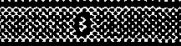 & 0 & 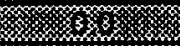 \\
\hline & & & & & m & & 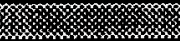 \\
\hline Wash Cycle & & & & & & & 桨要 \\
\hline Wash & st & 50 & 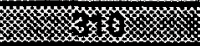 & 6490 & 极 & 12435 & 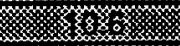 \\
\hline Add NaOH & (x) & 8 & 䃕 & 1246 & $x$ & 14217 & 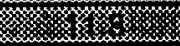 \\
\hline Wash & 答 & 70 & 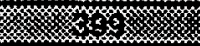 & 18099 & sy & 22613 & 的 \\
\hline Add $\mathrm{NaOH}$ & 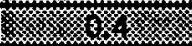 & 6 & sck & 1617 & 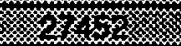 & 23267 & 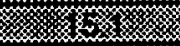 \\
\hline Wash & 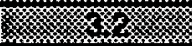 & 65 & 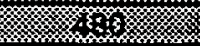 & 19906 & 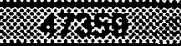 & 27963 & x \\
\hline Add NaNO2 & s. & 8 & sis & 2497 & s. & 28494 & 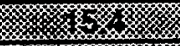 \\
\hline Wash & scis & 125 & 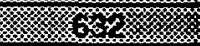 & 49055 & s. & 26751 & 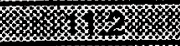 \\
\hline Sample & $x$ & - & 6 & 11662 & 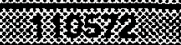 & 15090 & 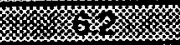 \\
\hline Transfer Slumy & zot & 130 & t & 2066 & s & 13023 & 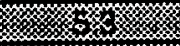 \\
\hline Stop & 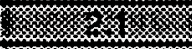 & - & 稵 & 3788 & 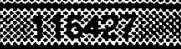 & 9235 & 答 \\
\hline
\end{tabular}

SS = Salt Solution

RWW = Recycled Wash Water

Italicized volume changes indicate removal from Tank 48.

Non-italicized volume changes indicate additions to Tank 48.

The wash cycle volume changes do not cause volume level changes in Tank 48 since the washwater and inhibitor solutions are added and removed simultaneously.

The volume of the contents in Tank 22 is incrementally increased by Late washwater at the rate of 2809 gallons per day. 


\section{APPENDIX B (continued)}

Concentration of TBP Added: TPB Solubility in DSS: TPB Solublitty in Wash Water: DSS TBP Hydrolysis Rate: TBP Hydrolysls Rate Constant:

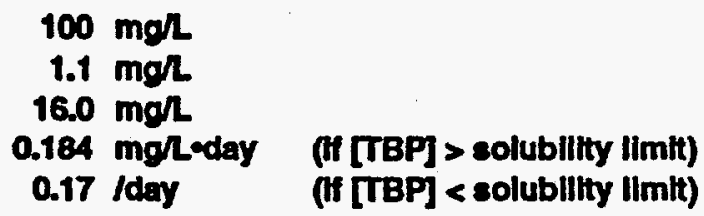

\begin{tabular}{|c|c|c|c|c|c|c|c|}
\hline \multicolumn{3}{|c|}{ Process } & \multicolumn{5}{|c|}{ Tank 50-Cycle 1} \\
\hline Step & 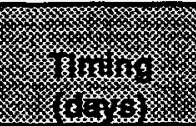 & $\begin{array}{l}\text { Volume } \\
\text { Change } \\
\text { (Kgal) }\end{array}$ & (k) & $\begin{array}{c}\text { TBP } \\
\text { Decomp. } \\
\text { (q) }\end{array}$ & 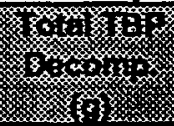 & $\begin{array}{c}\text { Remaining } \\
\text { TBP } \\
\text { (g) }\end{array}$ & 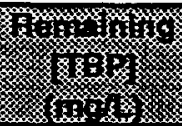 \\
\hline & X & & 位 & & & & 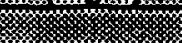 \\
\hline Batch *1 & & & s.s. & & & & \\
\hline Transfer SS & 䇣 & 630 & $x$ & & 1. & & 鷀 \\
\hline Transfer RWW & xy & 217 & x. & & 锿 & & 玹 \\
\hline Add Misc. Soln. & 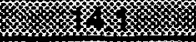 & 40.1 & . & & $1 \times$ & & 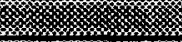 \\
\hline Filter to 7.5 wt \% & 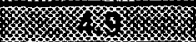 & 813.5 & 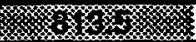 & & & $3.08 E+05$ & 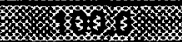 \\
\hline Filter to $10 \mathrm{wt} \%$ & 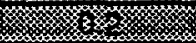 & 18.4 & . & & 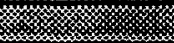 & $3.15 E+05$ & 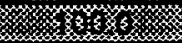 \\
\hline Stop/Start & - ty & - & 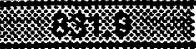 & & & $3.15 E+05$ & 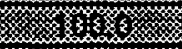 \\
\hline & & & 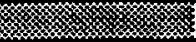 & & 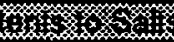 & ne & . \\
\hline Batch 2 & 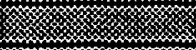 & & 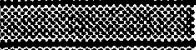 & & 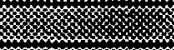 & & 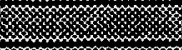 \\
\hline Transfer SS & 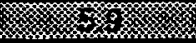 & 630 & m & & 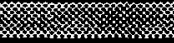 & & \\
\hline Transfer RWW & 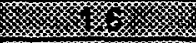 & 217 & 2 & & & & $\infty$ \\
\hline Add Misc. Soln. & 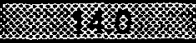 & 27 & . & & & & \\
\hline Filter to 7.5 wt \% & . & 806.7 & 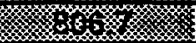 & & 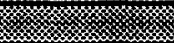 & $3.05 E+0.5$ & 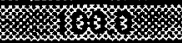 \\
\hline Filter to $10 w \%$ & 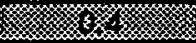 & 30.6 & 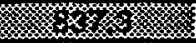 & & (1) & $3.17 E+05$ & 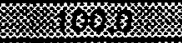 \\
\hline Stop/Stant & 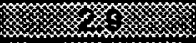 & - & 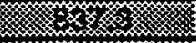 & & 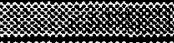 & $3.17 E+05$ & 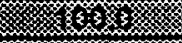 \\
\hline & 柆 & & 妶 & & 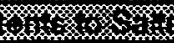 & ne & 筑 \\
\hline Batch *3 & & & & & 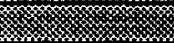 & & 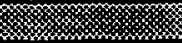 \\
\hline Transfer SS & 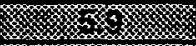 & 630 & . & & 呟 & & \\
\hline Transfer RWW & xy & 217 & 18 & & & & \\
\hline Add Misc. Soln. & . & 27 & 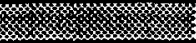 & & $x$ & & 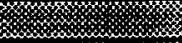 \\
\hline Filter to $7.5 w t \%$ & 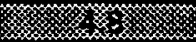 & 798.6 & mo & & & $3.02 E+05$ & 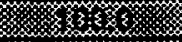 \\
\hline Filter to $10 \mathrm{wt} \%$ & 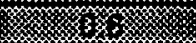 & 40.2 & 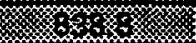 & & 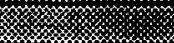 & $3.17 E+05$ & 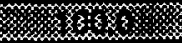 \\
\hline Stop/Stant & 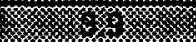 & $\overline{-}$ & 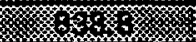 & & 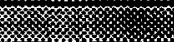 & $3.17 E+05$ & 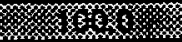 \\
\hline & & & & & (6) & ne & 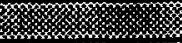 \\
\hline Wash Cycle & & & 2 & & & & sxix \\
\hline Wash & s. & 50 & 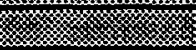 & & & & 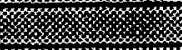 \\
\hline Add $\mathrm{NaOH}$ & 位 & 8 & (x) & & & & 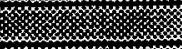 \\
\hline Wash & 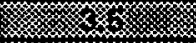 & 70 & 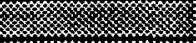 & & 畜 & & 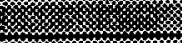 \\
\hline Add $\mathrm{NaOH}$ & m & 6 & . & & 媇 & & 妶 \\
\hline Wash & 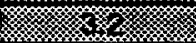 & 65 & m. & & ry & & 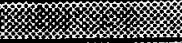 \\
\hline Add $\mathrm{NaNO}_{2}$ & 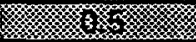 & 8 & 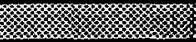 & & $2 x$ & & \\
\hline Wash & z & 125 & & & $\bar{x}$ & & (x) \\
\hline Sample & 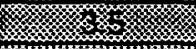 & - & 正 & & x & & 紧经 \\
\hline Transfer Slurry & 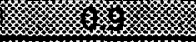 & 130 & 亦 & & 被纹 & & x \\
\hline Stop & 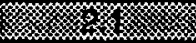 & - & 秝 & & 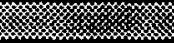 & & \\
\hline & 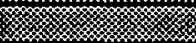 & & m & & 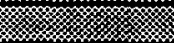 & & 鿆 \\
\hline
\end{tabular}

SS = Salt Solution

RWW = Recycled Wash Water

Atalicized volume changes indicate removal from Tank 48.

Non-italicized volume changes indicate additions to Tank-48.

The wash cycle volume changes do not cause volume level changes in Tank 48 since the washwater and inhibitor solutions are added and removed simultaneously.

The volume of the contents in Tank 22 is incrementally increased by Late washwater at the rate of 2809 gallons per day. 


\section{APPENDIX B (continued)}

Concentration of TBP Added: TPB Solublitty in DSS:

TPB Solublilty in Wash Water: DSS TBP Hydrolysls Rate: TBP Hydrolysis Rate Constant:
$100 \mathrm{mg} /$

$1.1 \mathrm{mgh}$

$16.0 \mathrm{mgh}$

$0.184 \mathrm{mgh}$-day (II [TBP] $>$ solubility limit)

0.17 iday (if [TBP] < solubility limit)

\begin{tabular}{|c|c|c|c|c|c|c|c|}
\hline \multicolumn{3}{|c|}{ Process } & \multicolumn{5}{|c|}{ Tank 48 - Cycle 2} \\
\hline Step & 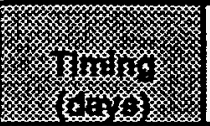 & $\begin{array}{l}\text { Volume } \\
\text { Change } \\
\text { (Kgal) }\end{array}$ & 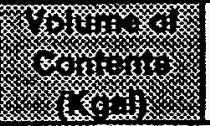 & $\begin{array}{c}\text { TBP } \\
\text { Decomp. } \\
\text { (g) }\end{array}$ & $\frac{1}{2}$ & $\begin{array}{c}\text { Remaining } \\
\text { TBP } \\
\text { (g) }\end{array}$ & (1) \\
\hline & $x_{x}$ & & . & & 氺 & & \\
\hline Batch 1 & 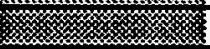 & & 媇 & & c & & x. \\
\hline Transfer SS & 慈然 & 630 & 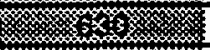 & 0 & 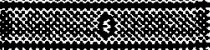 & 0 & 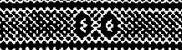 \\
\hline Transfer RWW & 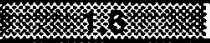 & 217 & 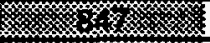 & 0 & 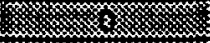 & 1859 & x \\
\hline Add Misc. Soln. & 媇媇 & 40.1 & 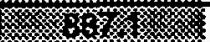 & 1677 & 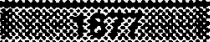 & 182 & (x) \\
\hline Filter to $7.5 \mathrm{wt} \%$ & 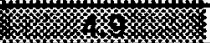 & 813.5 & - s. & 176 & (t) & 1 & 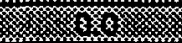 \\
\hline Filter to $10 \mathrm{wt} \%$ & (1) & 18.4 & . & 0 & 5 & 0 & (n) \\
\hline Stop/Start & $x$ & - & -2 & 0 & -6 & 0 & 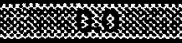 \\
\hline & 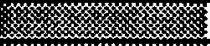 & & & & . & & \\
\hline Batch 12 & & & 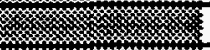 & & $\ldots$ & & 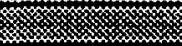 \\
\hline Transfer SS & 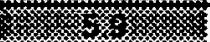 & 630 & 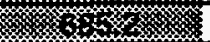 & 0 & 聯 & 0 & ( \\
\hline Transfer AWW & 怒 & 217 & xy & 0 & 等 & 9 & ston \\
\hline Add Misc. Soln. & 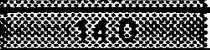 & 27 & 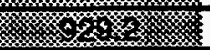 & 8 & 箖新 & 1 & 6 \\
\hline Filter to 7.5 wt \% & 筇 & 806.7 & 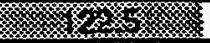 & 1 & 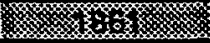 & 0 & 6 \\
\hline Filter to $10 \mathrm{wt} \%$ & 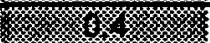 & 30.6 & m & $\overline{0}$ & (x) & 0 & 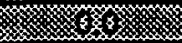 \\
\hline Stop/Start & 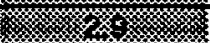 & - & 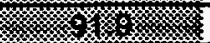 & 0 & 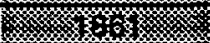 & 0 & 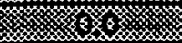 \\
\hline & 站 & & 桨 & & 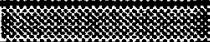 & & \\
\hline Batch * 3 & 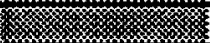 & & 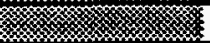 & & 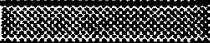 & & \\
\hline Transter SS & ry & 630 & 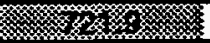 & 0 & 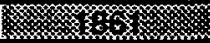 & 0 & 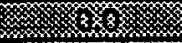 \\
\hline Transfer RWW & 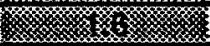 & 217 & . & $\overline{0}$ & 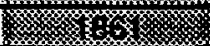 & 0 & 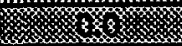 \\
\hline Add Misc. Soln. & W & 27 & \% & $\overline{0}$ & (x) & $\overline{0}$ & 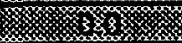 \\
\hline Filter to $7.5 \mathrm{wt} \%$ & s. & 798.6 & ct & $\overline{0}$ & 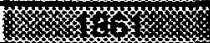 & $\overline{0}$ & s. \\
\hline Filter to $10 \mathrm{w} \%$ & I & 40.2 & 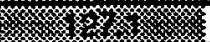 & $\overline{0}$ & 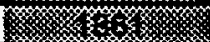 & 0 & 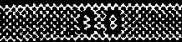 \\
\hline Stop/Stant & 㘳 & - & 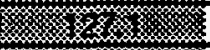 & 0 & 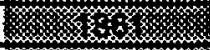 & 0 & 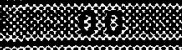 \\
\hline & (x) & & $\ldots$ & & 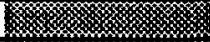 & & \\
\hline Wash Cycle & $x_{x}$ & & $x$ & & 举 & & 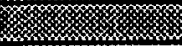 \\
\hline Wash & 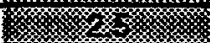 & 50 & 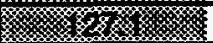 & 0 & 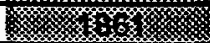 & $\overline{0}$ & (6) \\
\hline$\overline{\text { Add } \mathrm{NaOH}}$ & (4) & 8 & কy & $\overline{0}$ & ( & 0 & 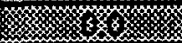 \\
\hline Wash & 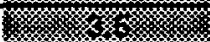 & 70 & 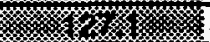 & 0 & 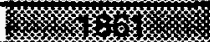 & 0 & 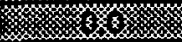 \\
\hline$\overline{\text { Add } \mathrm{NaOH}}$ & 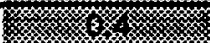 & 6 & $x$ & 0 & 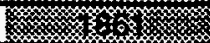 & $\overline{0}$ & 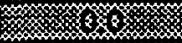 \\
\hline Wash & 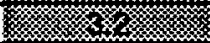 & 65 & 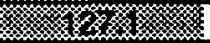 & 0 & 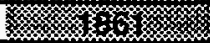 & $\mathbf{0}$ & 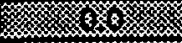 \\
\hline Add NaNO2 & 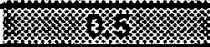 & 8 & $x_{x} x$ ck & 0 & 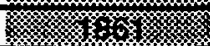 & $\overline{0}$ & 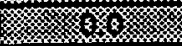 \\
\hline Wash & (l) & 125 & ( & 0 & (6) & $\overline{0}$ & 3 \\
\hline Sample & 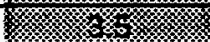 & - & 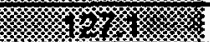 & 0 & 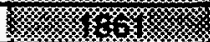 & 0 & 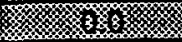 \\
\hline Transfer Slumy & W. & 130 & 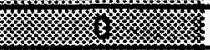 & 0 & $x_{x}$ & 0 & 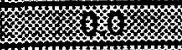 \\
\hline Stop & K & - & xy & 0 & K & 0 & t. \\
\hline & $y_{x}$ & & 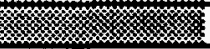 & & 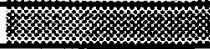 & & $x$ \\
\hline
\end{tabular}

SS = Salt Solution

RWW = Recycled Wash Water

thalicized volume changes indicate removal from Tank 48.

Non-italicized volume changes indicate additions to Tank 48 .

The wash cycle volume changes do not cause volume level changes in Tank 48 since the washwater and inhibitor solutions are added and removed simultaneousty.

The volume of the contents in Tank 22 is incrementally increased by Late washwater at the rate of 2809 gallons per day. 


\section{APPENDIX B (continued)}

\section{Concentration of TBP Added: TPB Solublitty in DSS: \\ TPB Solublilty in Wash Water: \\ RWW TBP Hydrolysls Rate: \\ TBP Hydrolysls Rate Constant:}

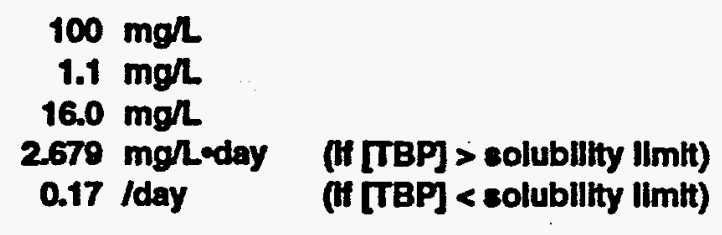

\begin{tabular}{|c|c|c|c|c|c|c|c|}
\hline \multicolumn{3}{|c|}{ Procese } & \multicolumn{5}{|c|}{ Tank 22 - Cycle 2} \\
\hline Step & , & $\begin{array}{l}\text { Volume } \\
\text { Change } \\
\text { (Kgal) }\end{array}$ & 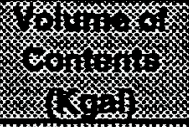 & $\begin{array}{c}\text { TBP } \\
\text { Decomp. } \\
\text { (g) }\end{array}$ & 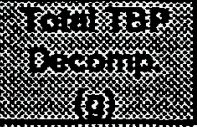 & $\begin{array}{c}\text { Remaining } \\
\text { TBP } \\
\text { (g) }\end{array}$ & 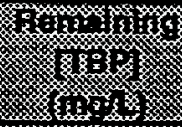 \\
\hline & & & > & & - & & \\
\hline Batch $\| 1$ & & & 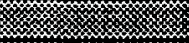 & & 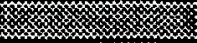 & & 玹 \\
\hline Transfer SS & 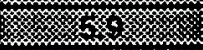 & 630 & 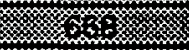 & 3517 & s & 5718 & … \\
\hline Transfer RWW & 缀 & 217 & 答 & 2739 & 留 & 2011 & 縈 \\
\hline Add Misc. Soln. & 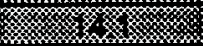 & 40.1 & 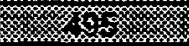 & 1806 & 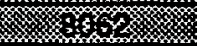 & 205 & . \\
\hline Filter to $7.5 w \%$ & 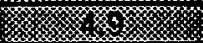 & 813.5 & 13 & 112 & 极 & 92 & 30 \\
\hline Filter to $10 m \%$ & 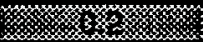 & 18.4 & zos & 3 & 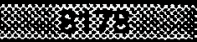 & 89 & ; \\
\hline Stop/Stant & 阵 & - & 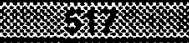 & 34 & 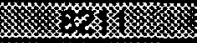 & 56 & 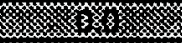 \\
\hline & & & 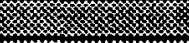 & & $x$ & & 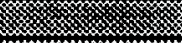 \\
\hline Batch $: 2$ & 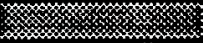 & & m & & 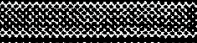 & & 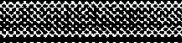 \\
\hline Transfer SS & 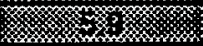 & 630 & x & 34 & 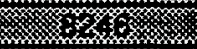 & 21 & 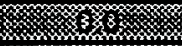 \\
\hline Transfer RWW & xyx & 217 & s. & 12 & 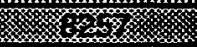 & 6 & 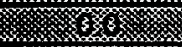 \\
\hline Add Misc. Soln. & (1) & 27 & 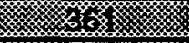 & 5 & 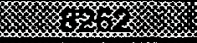 & 1 & 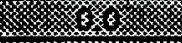 \\
\hline Filter to 7.5 wt \% & 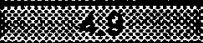 & 806.7 & s. & 0 & (4) & 0 & 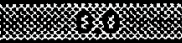 \\
\hline Filter to $10 \mathrm{wt} \%$ & W & 30.6 & -1 & 0 & - & 0 & 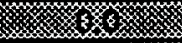 \\
\hline Stop/Start & 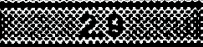 & $=$ & 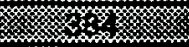 & $\overline{0}$ & x. & 0 & 好 \\
\hline & 项 & & 级 & & m & & 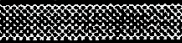 \\
\hline Batch $\# 3$ & 新 & & 将 & & 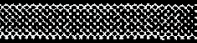 & & 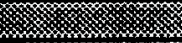 \\
\hline Transfer SS & 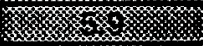 & 630 & w & $\mathbf{0}$ & mis & $\overline{0}$ & 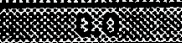 \\
\hline Transfer RWW & 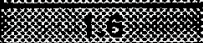 & 217 & 媇 & $\mathbf{0}$ & 460 & $\overline{0}$ & 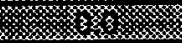 \\
\hline Add Misc. Soln. & 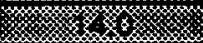 & 27 & \% & $\mathbf{0}$ & 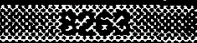 & $\begin{array}{l}0 \\
\end{array}$ & $3 \times 3$ \\
\hline Filter to $7.5 \mathrm{wt} \%$ & 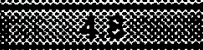 & 798.6 & 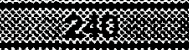 & 0 & 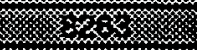 & 0 & 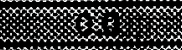 \\
\hline Filter to $10 \mathrm{w} \%$ & 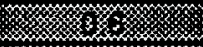 & 40.2 & 2 & 0 & 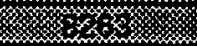 & $\overline{0}$ & 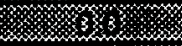 \\
\hline Stop/Start & mx & - & stis & 0 & 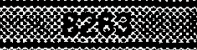 & 0 & 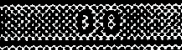 \\
\hline & & & & & & & 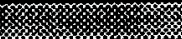 \\
\hline Wash Cycle & & & 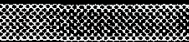 & & 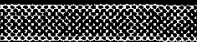 & & \\
\hline Wash & 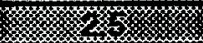 & 50 & 1 & 6490 & 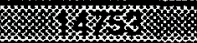 & 12435 & 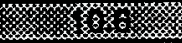 \\
\hline Add $\mathrm{NaOH}$ & 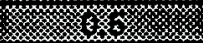 & 8 & 的 & 1246 & 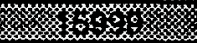 & 14217 & 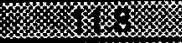 \\
\hline Wash & 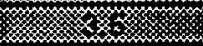 & 70 & xystos & 18099 & 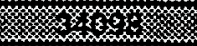 & 22613 & 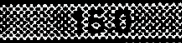 \\
\hline Add $\mathrm{NaOH}$ & 烈 & 6 & 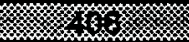 & 1617 & 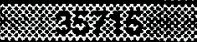 & 23267 & X) \\
\hline Wash & 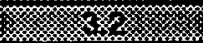 & 65 & 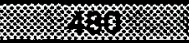 & 19906 & 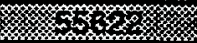 & 27963 & 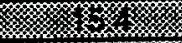 \\
\hline Add NaNO2 & 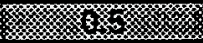 & 8 & 25 & 2497 & 58 & 28494 & s. \\
\hline Wash & k & 125 & 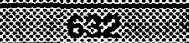 & 49055 & 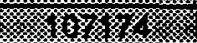 & 26751 & $x$ \\
\hline Sample & s. & - & 26 & 11662 & 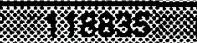 & 15090 & -2 \\
\hline Transfer Slurry & 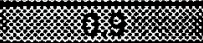 & 130 & 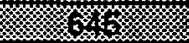 & 2066 & 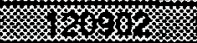 & 13023 & 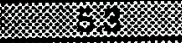 \\
\hline Stop & 的 & - & 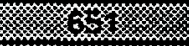 & 3788 & 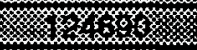 & 9235 & 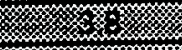 \\
\hline & 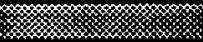 & & 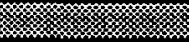 & & 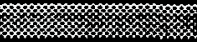 & & 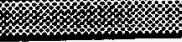 \\
\hline
\end{tabular}

SS = Salt Solution

RWW = Recycled Wash Water

Italicized volume changes indicate removal from Tank 48.

Non-italicized volume changes indicate additions to Tank 48.

The wash cycle volume changes do not cause volume level changes in Tank 48 since the washwater and inhibitor solutions are added and removed simultaneously.

The volume of the contents in Tank 22 is incrementally increased by Late washwater at the rate of 2809 gallons per day. 
APPENDIX B (continued)

\section{Concentration of TBP Added: TPB Solubllity in DSS: TPB Solubility in Wash Water: DSS TBP Hydrolysle Rate: TBP Hydrolysle Rate Constant:}

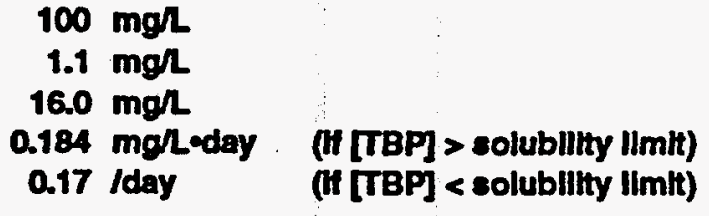

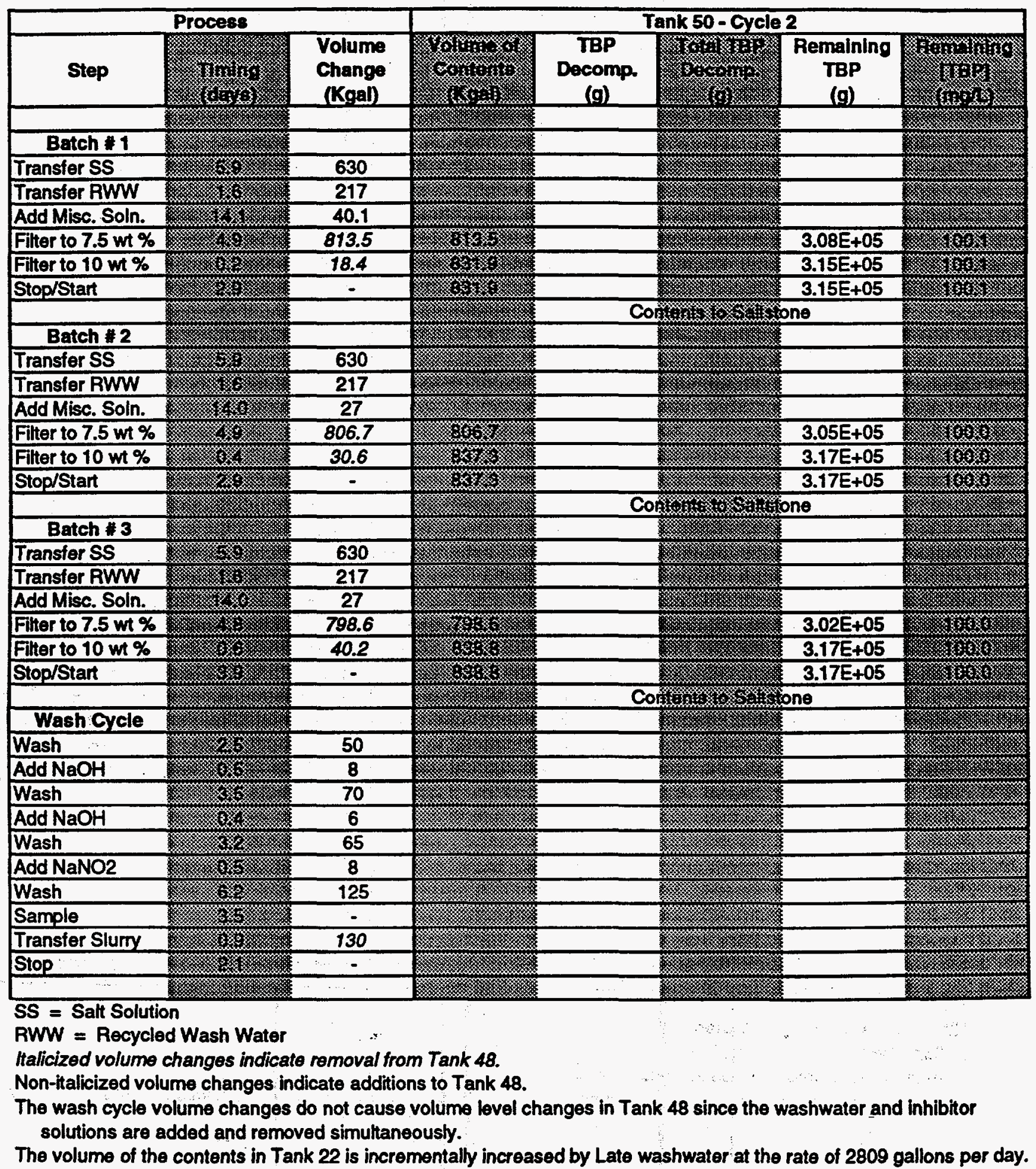




\section{APPENDIX B (continued)}

\section{Concentration of TBP Added: TPB Solubllity in DSS: \\ TPB Solubllity in Wach Water: \\ DSS TBP Hydrolysls Rate: \\ TBP Hydrolysis Rate Constant:}

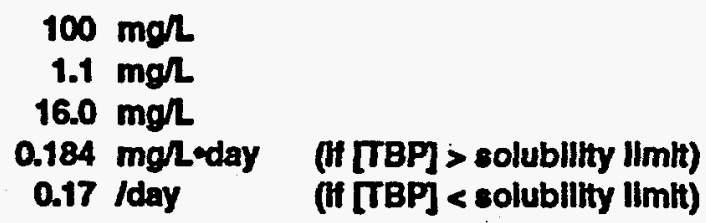

\begin{tabular}{|c|c|c|c|c|c|c|c|}
\hline \multicolumn{3}{|c|}{ Process } & \multicolumn{5}{|c|}{ Tank 48-Cycle 3} \\
\hline Step & 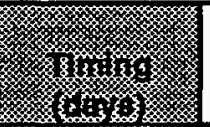 & $\begin{array}{l}\text { Volume } \\
\text { Change } \\
\text { (Kgal) }\end{array}$ & 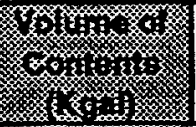 & $\begin{array}{c}\text { TBP } \\
\text { Decomp. } \\
\text { (g) }\end{array}$ & 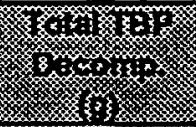 & $\begin{array}{c}\text { Remaining } \\
\text { TBP } \\
\text { (g) }\end{array}$ & 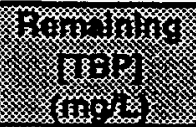 \\
\hline & (1) & & . & & (x) & & \\
\hline Batch 1 & 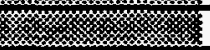 & & 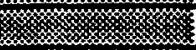 & & & & 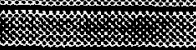 \\
\hline Transfer SS & s. & 630 & 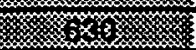 & 0 & $x$ & 0 & 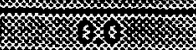 \\
\hline Transfer RWW & x & 217 & 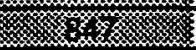 & 0 & $x$ & 1859 & 燚 \\
\hline Add Misc. Soin. & 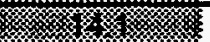 & 40.1 & 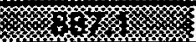 & 1677 & 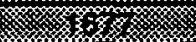 & 182 & 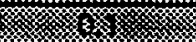 \\
\hline Filter to 7.5 wt \% & 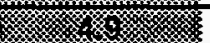 & 813.5 & xy & 176 & 致 & $T$ & X \\
\hline Filter to $10 w \%$ & twis & 18.4 & (3) & $\overline{0}$ & xys & $\overline{0}$ & 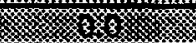 \\
\hline Stop/Start & (x) & - & s. & 0 & 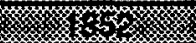 & $\overline{0}$ & k3. \\
\hline & 稡 & & 1. & & 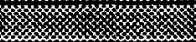 & & $\ldots$ \\
\hline Batch $\# 2$ & 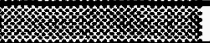 & & 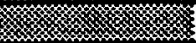 & & 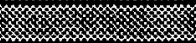 & & \\
\hline Transfer SS & 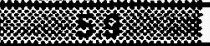 & 630 & my & 0 & 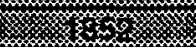 & $\overline{0}$ & m \\
\hline Transfer RWW & 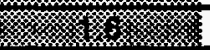 & 217 & $x$ & 0 & $x$ & 9 & s. \\
\hline Add Misc. Soln. & 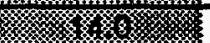 & 27 & 45 & 8 & 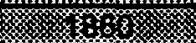 & $T$ & (3) \\
\hline Filter to $7.5 \mathrm{wt} \%$ & 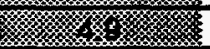 & 806.7 & w & 1 & 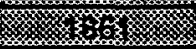 & 0 & z \\
\hline Filter to $10 \mathrm{w} \%$ & $x$ & 30.6 & xy & 0 & x & 0 & zx \\
\hline Stop/Stant & 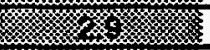 & - & - & 0 & s. & 0 & 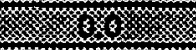 \\
\hline & 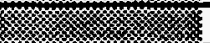 & & 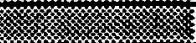 & & m & & 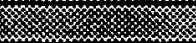 \\
\hline Batch \# 3 & m & & (x) & & & & \\
\hline Transfer SS & wa & 630 & 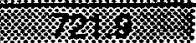 & $\overline{0}$ & 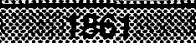 & $\overline{0}$ & 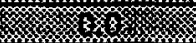 \\
\hline Transfer RWW & \% & 217 & \% & 0 & 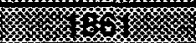 & 0 & 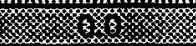 \\
\hline Add Misc. Soln. & $x$ & 27 & 86 & 0 & rescos & 0 & S3: \\
\hline Filter to $7.5 \mathrm{wt} \%$ & 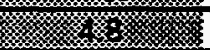 & 798.6 & x & 0 & 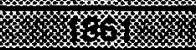 & 0 & s. \\
\hline Filter to $10 \mathrm{wt} \%$ & 列等 & 40.2 & 至 & 0 & 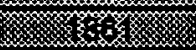 & 0 & 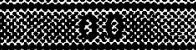 \\
\hline Stop/Start & 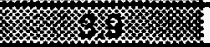 & $=$ & 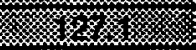 & 0 & 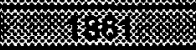 & 0 & (6) \\
\hline & 沙 & & & & & & 泣 \\
\hline Wash Cycle & 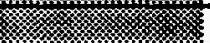 & & (1) & & 列 & & 纹 \\
\hline Wash & 2 & 50 & xy & 0 & 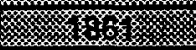 & 0 & . \\
\hline Add $\mathrm{NaOH}$ & (6) & 8 & X. & 0 & 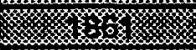 & 0 & 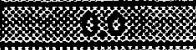 \\
\hline Wash & 经 & 70 & 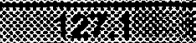 & 0 & 4 & 0 & 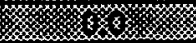 \\
\hline Add $\mathrm{NaOH}$ & (6) & 6 & kn & $\overline{0}$ & $x$ & $\overline{0}$ & 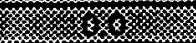 \\
\hline Wash & 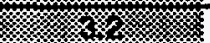 & 65 & (4) & $\overline{0}$ & \% & 0 & 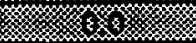 \\
\hline Add NaNO2 & 䇣 & 8 & 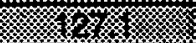 & 0 & 管 & 0 & ry \\
\hline Wash & 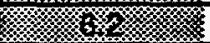 & 125 & 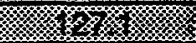 & 0 & 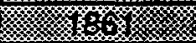 & 0 & s. \\
\hline Sample & sis & - & 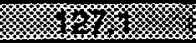 & 0 & 要 & 0 & 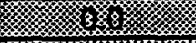 \\
\hline Transfer Slurry & 6 & 130 & (1) & 0 & 66 & 0 & $\frac{1}{3563}$ \\
\hline Stop & tis & - & 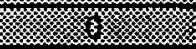 & 0 & 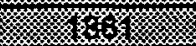 & 0 & 䇣新 \\
\hline & ) & & $m$ & & 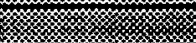 & & 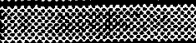 \\
\hline
\end{tabular}

SS = Salt Solution

RWW = Recycled Wash Water

Italicized volume changes indicate removal from Tank 48.

Non-italicized volume changes indicate additions to Tank 48.

The wash cycle volume changes do not cause volume level changes in Tank 48 since the washwater and inhibitor solutions are added and removed simultaneously.

The volume of the contents in Tank 22 is incrementally increased by Late washwater at the rate of $2 \overline{8} 09$ gallons per day. 
APPENDIX B (continued)

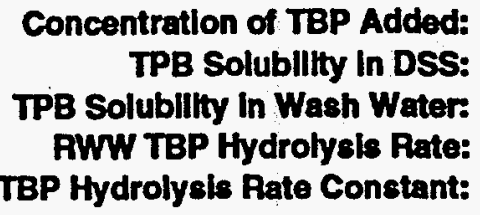

\author{
$100 \mathrm{mgh}$ \\ $1.1 \mathrm{mgh}$ \\ $16.0 \mathrm{mgl}$ \\ $2.679 \mathrm{mgh}$-day \\ $0.17 / d a y$
}

(if [TBP] > solubillty limit)

(If [TBP] < solubility limt)

\begin{tabular}{|c|c|c|c|c|c|c|c|}
\hline \multicolumn{3}{|c|}{ Process } & \multicolumn{5}{|c|}{ Tank 22 - Gycle 3} \\
\hline Step & $\ln (x)$ & $\begin{array}{l}\text { Volume } \\
\text { Change } \\
\text { (Kgal) }\end{array}$ & ons & $\begin{array}{c}\text { TBP } \\
\text { Decomp. } \\
\text { (g) }\end{array}$ & (3) & $\begin{array}{c}\text { Remaining } \\
\text { TBP } \\
\text { (g) }\end{array}$ & 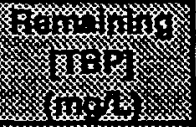 \\
\hline & 媇 & & 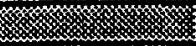 & & (1) & & 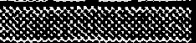 \\
\hline Batch 1 & $x$ & & 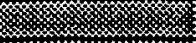 & & 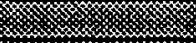 & & 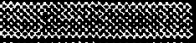 \\
\hline Transter SS & 格 & 630 & 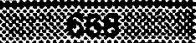 & 3517 & - & 5718 & x \\
\hline Transter RWW & 筮 & 217 & 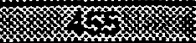 & 2739 & 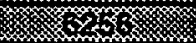 & 2011 & m \\
\hline Add Misc. Soln. & 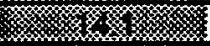 & 40.1 & $x$ & 1806 & . & 205 & $x$ \\
\hline Filter to $7.5 w t \%$ & 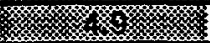 & 813.5 & 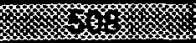 & 112 & . & 92 & 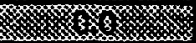 \\
\hline Filter to $10 \mathrm{wt} \%$ & x & 18.4 & zot & 3 & 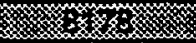 & 89 & 给 \\
\hline Stop/Start & 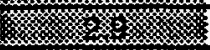 & - & 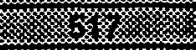 & 34 & w & 56 & th \\
\hline & & & . & & 杪 & & 氺 \\
\hline Batch 2 & 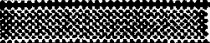 & & 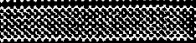 & & 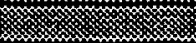 & & \\
\hline Transfer SS & $x$ & 630 & x. & 34 & s. & 21 & m \\
\hline Transfer RWW & was & 217 & - & 12 & sins & 6 & 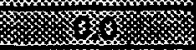 \\
\hline Add Misc. Soln. & X) & 27 & 6 & 5 & 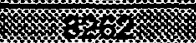 & 1 & 傩 \\
\hline Filter to $7.5 w t \%$ & 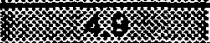 & 806.7 & s & $\overline{0}$ & scts & 0 & 烈 \\
\hline Filter to $10 \mathrm{wt} \%$ & (1) & 30.6 & 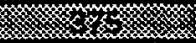 & 0 & 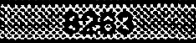 & 0 & 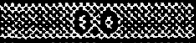 \\
\hline Stop/Start & $\psi_{x}$ & - & x. & 0 & w & 0 & 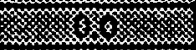 \\
\hline & & & 媇 & & 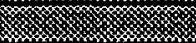 & & $x_{x}$ \\
\hline Batch \# 3 & 媇 & & 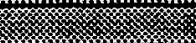 & & $x$ & & 准 \\
\hline Transfer SS & 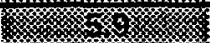 & 630 & 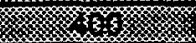 & $\overline{0}$ & xis. & 0 & 16x \\
\hline Transfer RWW & . & 217 & $x$ & 0 & 35 & 0 & s. \\
\hline Add Misc. Soln. & K) & 27 & 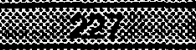 & 0 & x & 0 & 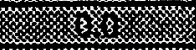 \\
\hline Filter to $7.5 \mathrm{wt} \%$ & 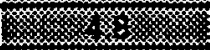 & 798.6 & . & 0 & 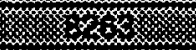 & 0 & 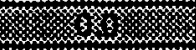 \\
\hline Filter to $10 \mathrm{wt} \%$ & 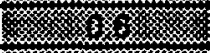 & 40.2 & 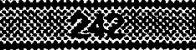 & 0 & 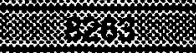 & 0 & 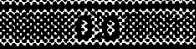 \\
\hline Stop/Start & $x$ & - & $x$ & 0 & 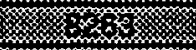 & 0 & 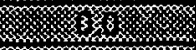 \\
\hline & 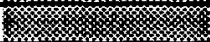 & & 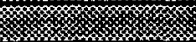 & & 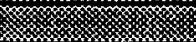 & & 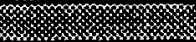 \\
\hline Wash Cycle & & & & & x & & 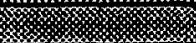 \\
\hline Wash & (5) & 50 & 4 & 6490 & m & 12435 & 36 \\
\hline Add $\mathrm{NaOH}$ & 玹 & 8 & 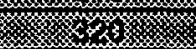 & 1246 & 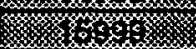 & 14217 & 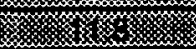 \\
\hline Wash & Xx & 70 & 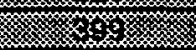 & 18099 & 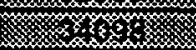 & 22613 & 好 \\
\hline Add $\mathrm{NaOH}$ & 3 & 6 & 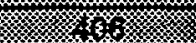 & 1617 & 学楼 & 23267 & 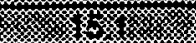 \\
\hline Wash & 网 & 65 & 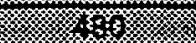 & 19906 & 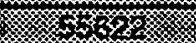 & 27963 & 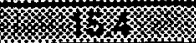 \\
\hline Add NaNO2 & xy & 8 & $\frac{1}{25}$ & 2497 & s. & 28494 & 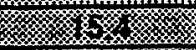 \\
\hline Wash & x & 125 & 6 & 49055 & w & 26751 & (1) \\
\hline Sample & K & - & 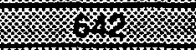 & 11662 & X & 15090 & x. \\
\hline Transfer Slumy & . & 130 & s. & 2066 & 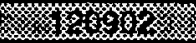 & 13023 & 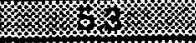 \\
\hline Stop & $x_{x}$ & - & 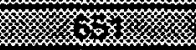 & 3788 & 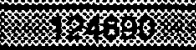 & 9235 & 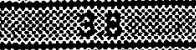 \\
\hline & 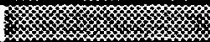 & & 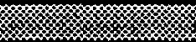 & & 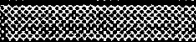 & & 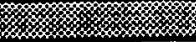 \\
\hline
\end{tabular}

SS = Salt Solution

RWW = Recycled Wash Water

Halicized volume changes indicate removal from Tank 48.

Non-italicized volume changes indicate additions to Tank 48.

The wash cycle volume changes do not cause volume level changes in Tank 48 since the washwater and inhibitor solutions are added and removed simultaneously.

The volume of the contents in Tank 22 is incrementally increased by Late washwater at the rate of 2809 gallons per day. 


\section{APPENDIX B (continued)}

Concentration of TBP Added: TPB Solublitty in DSS:

TPB Solublilty in Wash Water:

DSS TBP Hydrolyels Rate:

TBP Hydrolysis Rate Constant:

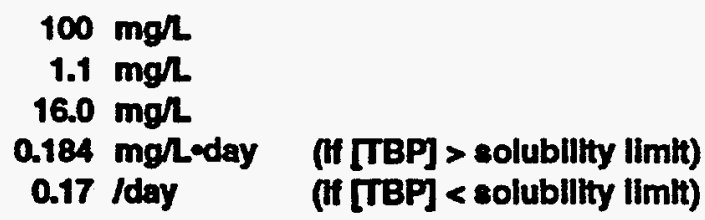

$100 \mathrm{mgh}$

$1.1 \mathrm{mgh}$

$16.0 \mathrm{mg} n$

$0.184 \mathrm{mg}$ Leday (if [TBP] > solublilty limit)

0.17 /day (If [TBP] < solubllity limit)

\begin{tabular}{|c|c|c|c|c|c|c|c|}
\hline \multicolumn{3}{|c|}{ Process } & \multicolumn{5}{|c|}{ Tank 50 - Cycle 3} \\
\hline Step & 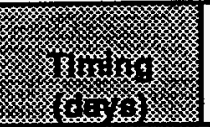 & $\begin{array}{c}\text { Volume } \\
\text { Change } \\
\text { (Kgal) }\end{array}$ & (3) & $\begin{array}{c}\text { TBP } \\
\text { Decomp. } \\
\text { (g) }\end{array}$ & , & $\begin{array}{c}\text { Remaining } \\
\text { TBP } \\
\text { (g) }\end{array}$ & 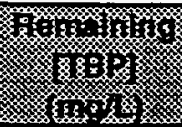 \\
\hline & . & & $x$ & & $\ldots$ & & . \\
\hline Batch $\div 1$ & & & 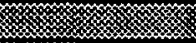 & & man & & 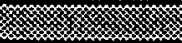 \\
\hline Transfer SS & $x$ & 630 & 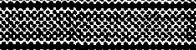 & & xy & & m \\
\hline Transfer RWW & 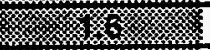 & 217 & 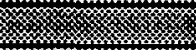 & & x妶 & & $x$ \\
\hline Add Misc. Soln. & 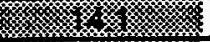 & 40.1 & . & & 绞 & $:$ & 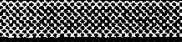 \\
\hline Filter to 7.5 wt \% & 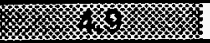 & 813.5 & . & & 妶 & $3.08 E+05$ & H \\
\hline Filter to $10 \mathrm{wt} \%$ & s. & 18.4 & - b. & & . & $3.15 E+05$ & 好的 \\
\hline Stop/Start & . & - & 氺 & & \% & $3.15 E+05$ & (1) \\
\hline & & & $x$ & & 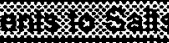 & one & sx \\
\hline Batch $: 2$ & m & & 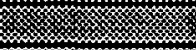 & & x & & \\
\hline Transfer SS & xys & 630 & y.sy & & 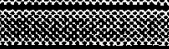 & & x. \\
\hline Transfer RWW & 6 & 217 & > & & - & & 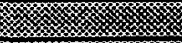 \\
\hline Add Misc. Soln. & 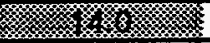 & 27 & & & & & 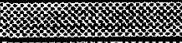 \\
\hline Filter to $7.5 \mathrm{wt} \%$ & 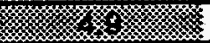 & 806.7 & \% & & 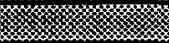 & $3.05 E+05$ & 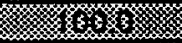 \\
\hline Filter to $10 \mathrm{wt} \%$ & 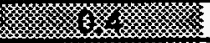 & 30.6 & 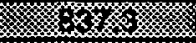 & & 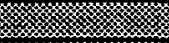 & $3.17 E+05$ & 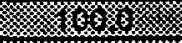 \\
\hline Stop/Start & 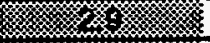 & - & 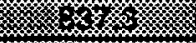 & & & $3.17 E+05$ & 14 \\
\hline & 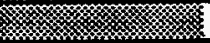 & & m & & mots & one & 准 \\
\hline Batch \# 3 & & & $8 x$ & & & & m \\
\hline Transier SS & x. & 630 & $\alpha$ & & 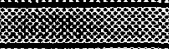 & & \\
\hline Transfer RWW & 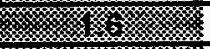 & 217 & & & KF & & 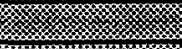 \\
\hline Add Misc. Soln. & 4 & 27 & 1. & & 伭 & & \\
\hline Filter to 7.5 wt $\%$ & 媇 & 798.6 & 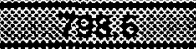 & & & $3.02 E+05$ & 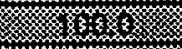 \\
\hline Filter to $10 w t \%$ & 燃 & 40.2 & 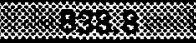 & & 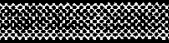 & $3.17 E+05$ & 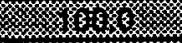 \\
\hline Stop/Start & 的的新 & - & 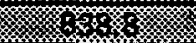 & & 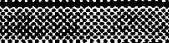 & $3.17 E+05$ & 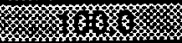 \\
\hline & & & & & H. & one & 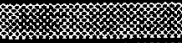 \\
\hline Wash Cycle & kx & & স্য় & & 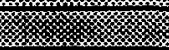 & & 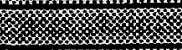 \\
\hline Wash & 委 & 50 & & & & & 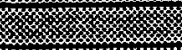 \\
\hline Add $\mathrm{NaOH}$ & 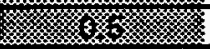 & 8 & $x$ & & 媇 & & strs \\
\hline Wash & 亲 & 70 & & & 媇 & & 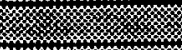 \\
\hline Add $\mathrm{NaOH}$ & m & 6 & 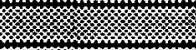 & & $\ldots$ & & rm \\
\hline Wash & 筮察察 & 65 & 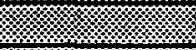 & & 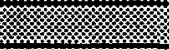 & & \\
\hline Add NaNO2 & 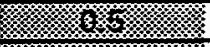 & 8 & צ & & W & & \\
\hline Wash & 6 & 125 & 筡 & & 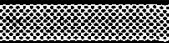 & & \\
\hline Sample & 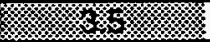 & - & X. & & r. & & k \\
\hline Transfer Slumy & 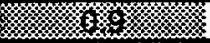 & 130 & 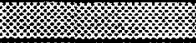 & & 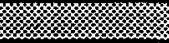 & & \\
\hline Stop & 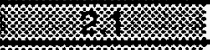 & - & $x$ & & 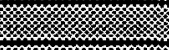 & & 窈聯 \\
\hline & x & & 两 & & স্যো & & \\
\hline
\end{tabular}

SS = Salt Solution

RWW = Recycled Wash Water

thalicized volume changes indicate removal from Tank 48.

Non-italicized volume changes indicate additions to Tank 48 .

The wash cycle volume changes do not cause volume level changes in Tank 48 since the washwater and inhibitor solutions are added and removed simultaneously.

The volume of the contents in Tank 22 is incrementally increased by Late washwater at the rate of 2809 gallons per day. 


\section{APPENDIX C}

Flammability calculations conceming 1-butanol in ITP facilities.

\section{Tank 22}

Use worst case conditions for basis:

- TBP is added at a concentration of $100 \mathrm{mg} /$. The total amount of TBP added to Tank 22 is $125 \mathrm{~kg}$ per cycle. (TBP will likely be injected at a maximum concentration of $40 \mathrm{mg} /$ ).

- The volume of wash water contained in Tank 22 is at its maximum (668 $\mathrm{kgal}$ based upon the material balance) resulting in the minimum volume of vapor space. (The volume of wash water in Tank 22 is expected to range between 188 and $668 \mathrm{kgal}$ per cycle).

- TBP decomposes completely to 1-butanol and phosphate whereby each hydrolysis step occurs at the same rate (conservative since DBP and MBP hydrolyze at a slower rate than TBP).

... Therefore, 3 times as many moles of 1 butanol are formed per mole of TBP decomposed over any given period of time.

- The maximum rate of TBP decomposition occurs when the TBP concentration is equal to or greater than its solubility limit.

- All 1 -butanol which is produced is vaporized instantaneously. (The solubility of 1-butanol in water is $7.35 \mathrm{~g} / 100 \mathrm{~g}$ and 1 -butanol will likely be soluble to a similar extent in wash water). ${ }^{33}$

Relevant information:

- The LFL for 1-butanol is $1.45 \%$ in air. 30

- Tank 22 is ventilated with air at a nominal rate of $300 \mathrm{scfm}(8500 \mathrm{Vmin}) .{ }^{34}$

- The first-order rate constant, $k$, for the hydrolysis of TBP is $0.007 \mathrm{hr}^{-1}\left(0.17\right.$ day $\left.^{-1}\right) .^{18}$

Rate of TBP hydrolysis for concentrations $\geq 16.0 \mathrm{mg} /$ (solubility limit):

$$
\begin{aligned}
\ln \frac{[\mathrm{TBP}]}{[\mathrm{TBP}]_{0}} & =-k t \\
{[\mathrm{TBP}] } & =[\mathrm{TBP}]_{0} e^{-k t} \\
{[\mathrm{TBP}] } & =(16.0) \mathrm{e}^{-.007(1 \mathrm{hr})} \\
{[\mathrm{TBP}] } & =15.89 \mathrm{mg} / \mathrm{L} \\
\Delta[\mathrm{TBP} \text { time } & =(16.0-15.89) / 1 \mathrm{hr} \\
\text { rate of TBP hydrolysis } & =0.11 \mathrm{mg} / \mathrm{soln} \cdot \mathrm{hr}
\end{aligned}
$$


Volume \% 1-butanol produced per minute:

$$
\begin{gathered}
\frac{0.11 \mathrm{mg}}{\text { L soln } \cdot \mathrm{hr}} \times \frac{1 \mathrm{~g}}{1000 \mathrm{mg}} \times \frac{1 \mathrm{~mole} \text { TBP }}{266.3 \mathrm{~g} \mathrm{TBP}} \times \frac{3 \text { moles 1-butanol }}{1 \text { mole TBP }} \times \frac{1 \mathrm{hr}}{60 \mathrm{~min}}= \\
=2.1 \times 10^{-8} \text { moles } 1 \text {-butanol } / \mathrm{L} \text { solnomin }
\end{gathered}
$$

Using the Ideal Gas Law, PV = nAT, allows for the conversion of moles 1-butanol produced per minute to volume of 1-butanol produced per minute.

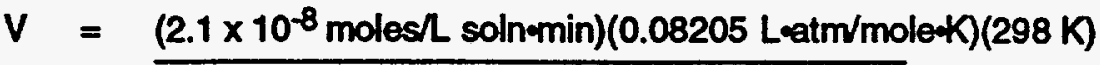

$$
\begin{aligned}
& 1 \text { atm } \\
& V=5.1 \times 10^{-7} \mathrm{~L} \text { 1-butanol } / \mathrm{L} \text { soln } \bullet \text { min }
\end{aligned}
$$

Assuming Tank 22 contains $668 \mathrm{kgal}(2,528,000 \mathrm{~L})$ of wash water, the amount of 1 -butanol produced per minute would be:

$$
\begin{aligned}
& V=\left(5.1 \times 10^{-7} \mathrm{~L} 1 \text {-butanol } / \mathrm{L} \text { soln } \cdot \min \right)(2,528,000 \mathrm{~L} \text { soln }) \\
& V=1.3 \mathrm{~L} 1 \text {-butanol } / \mathrm{min}
\end{aligned}
$$

Given the ventilation air flow of Tank 22 is $8500 \mathrm{~L} / \mathrm{min}$, then the volume \% of 1-butanol capable of being produced would be:

$$
\begin{aligned}
& \text { Volume \% 1-butanol }=\frac{1.3 \mathrm{~L} 1 \text {-butand } / \mathrm{min}}{8500 \mathrm{~L} \text { air } / \mathrm{min}} \times 100 \% \\
& \text { Volume } \% 1 \text {-butanol }=0.015 \%
\end{aligned}
$$

This is $1.0 \%$ of the LFL for 1 butanol. Based upon the rate of volume of 1 -butanol produced per minute, the time to reach LFL would be 18.5 days in the event ventilation was lost. 
Distribution:

8601

$\| 1001 / 5$

G. T. Wright, 5002H

H. M. Handfinger, $5002 \mathrm{H}$

J. P. Morin, 719-4A

R. G. Croley, 241-12OH

R. K. Roosa, 241-121H

J. N. Brooke, 241-119H

R. A. Scaggs, 703-H

W. B. Van Pelt, 241-152H

C. L. Leung, 241-154H

J. E. Marra, 703-H

M. C. Chandler, 703-H

D. A. Pervis, 241-121H

G. K. Georgeton, 703-H

T. P. Gaughan, 241-152H

W. L. Tamosaitis, 773-A

M. K. Gupta, 992W-1

J.P. Bibler, 773-A

D. D. Walker, 773-A

D. T. Hobbs, 773-A

R. F. Swingle, 773-A

J. F. McGlynn, 676-T

D. J. McCabe, 676-T

L. L. Kilpatrick, 676-T

M. L. Meyer, 676-1T

R. H. Hsu, 773-43A

C. A. Langton, $773-43 \mathrm{~A}$

L. F. Landon, 704-T

J. C. Marek, 704-T

M. A. Baich, 704-T

WT-LWG Files, 773-A

TIM, 703-43A

YVONNE

$\lg m$

Folewn-301.8 